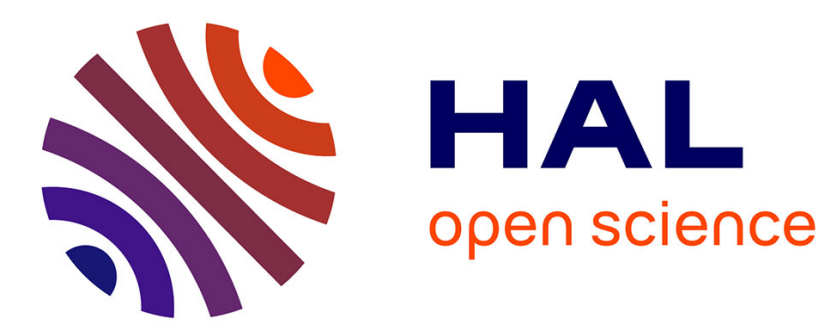

\title{
Self-sustained oscillations in variable-density round jets
}

J.W. Nichols, Peter J. Schmid, J.J. Riley

\section{To cite this version:}

J.W. Nichols, Peter J. Schmid, J.J. Riley. Self-sustained oscillations in variable-density round jets. Journal of Fluid Mechanics, 2007, 582 (july), pp.341-376. 10.1017/s0022112007005903 . hal01023338

\section{HAL Id: hal-01023338 \\ https://hal-polytechnique.archives-ouvertes.fr/hal-01023338}

Submitted on 20 Jul 2014

HAL is a multi-disciplinary open access archive for the deposit and dissemination of scientific research documents, whether they are published or not. The documents may come from teaching and research institutions in France or abroad, or from public or private research centers.
L'archive ouverte pluridisciplinaire HAL, est destinée au dépôt et à la diffusion de documents scientifiques de niveau recherche, publiés ou non, émanant des établissements d'enseignement et de recherche français ou étrangers, des laboratoires publics ou privés. 


\title{
Self-sustained oscillations in variable-density round jets
}

\author{
JOSEPH W. NICHOLS ${ }^{1} \dagger$, PETER J. SCHMID 2 \\ AND JAMES J. RILE Y \\ ${ }^{1}$ Department of Mechanical Engineering, University of Washington, Seattle, WA 98195, USA \\ ${ }^{2}$ Laboratoire d'Hydrodynamique (LadHyX), CNRS-École Polytechnique, 91128 Palaiseau, France
}

(Received 5 May 2006 and in revised form 23 January 2007)

The stability properties of round variable-density low-Mach-number jets are studied by means of direct numerical simulation (DNS) and linear stability analysis. Fully three-dimensional DNS of variable-density jets, with and without gravity, demonstrate that the presence of buoyancy causes a more abrupt transition to turbulence. This effect helps to explain differences between normal gravity and microgravity jet diffusion flames observed in the laboratory.

The complete spectrum of spatial eigenmodes of the linearized low-Mach-number equations is calculated using a global matrix method. Also, an analytic form for the continuous portion of this spectrum is derived, and used to verify the numerical method. The absolute instability of variable-density jets is confirmed using Brigg's method, and a comprehensive parametric study of the strength and frequency of this instability is performed. Effects of Reynolds number, the density ratio of ambientto-jet fluid $\left(S_{1}\right)$, shear-layer thickness and Froude number are considered. Finally, a region of local absolute instability is shown to exist in the near field of the jet by applying linear stability analysis to mean profiles measured from DNS.

\section{Introduction}

\subsection{Motivations}

A primary motivation for this work is to explain the marked differences between microgravity jet flames and normal gravity jet flames observed in drop-tower experiments performed by Bahadori et al. (1992) and Hegde, Zhou \& Bahadori (1994). In these experiments, which were repeated for different fuel types (methane, propylene and propane), transitional and turbulent microgravity flames were observed to be wider and much taller than corresponding normal gravity flames. Also, the normal gravity flame length reached a constant value in the limit of high Reynolds number, but the microgravity flame length appeared to increase monotonically with respect to Reynolds number. In the turbulent regime, for example, a microgravity flame might be twice as long as its normal gravity counterpart.

Bahadori et al. (1992) and Hegde et al. (1994) suggest that many of the observed differences between microgravity and normal gravity jet flames might be explained through changing jet stability properties. As we will discuss in further detail in this 
paper, many of these properties can be understood by considering the simpler problem of a non-reacting, but variable-density, jet. There is a large body of work available on the stability of variable-density jets (see Monkewitz \& Sohn 1988), but the theory traditionally neglects the effect of buoyancy. For jet diffusion flames, however, buoyancy appears to have a significant effect on the overall stability of the flow. It is not clear, though, whether buoyancy induced by heat release or buoyancy arising from a non-unity ratio of the density of air to that of cold fuel, $S_{1}$, plays a larger role in determining the overall stability properties of the flame. The latter effect is present in many combustion applications in which the density of the fuel does not match the density of air (for example, the density of methane is about half that of air at the same temperature). Furthermore, from an inviscid linear stability analysis, Monkewitz \& Sohn (1988) determined that variable density jets with $S_{1}>1.39$ are absolutely unstable, and support self-sustaining oscillations. As we will see, this absolute instability plays a key role in the near-field development of variable density jets. In the analysis of Monkewitz \& Sohn (1988), however, the jet was assumed to be dominated by inertia, so that the effect of buoyancy on this absolute instability was not considered. For these reasons, we restrict ourselves in this paper to considering only the influence of buoyancy induced by non-unity density ratio $S_{1}$, and thus consider only non-reacting variable-density jets. Nevertheless, at various points in this paper, we will refer back to the reacting problem and compare our results to observations of diffusion flames when appropriate.

Idicheria, Boxx \& Clemens (2003) repeated and extended some of the same droptower experiments. They did not, however, observe the dramatic increase of flame length under conditions of microgravity, as was observed by Bahadori et al. (1992) and Hegde et al. (1994). For all three fuels that Idicheria et al. (2003) studied (methane, ethylene and propane), the flame lengths of the microgravity flames behaved similarly to the flame lengths of the normal gravity flames for a large range of Reynolds number. They attribute the difference of their observations from those of previous workers to either the added presence of a pilot flame keeping the flame attached, or to the microgravity flames being more sensitive to external disturbances and thus more dependent on boundary conditions. These two seemingly opposing results of similar laboratory experiments provide further motivation for studying the fundamental fluid mechanics of the problem in more detail, separate from the complicating influence of the reactive terms.

\subsection{Previous work}

Through linear stability theory, Batchelor \& Gill (1962) demonstrated that a cylindrical vortex sheet in an inviscid incompressible fluid of uniform density is linearly unstable with respect to small disturbances of all azimuthal wavenumbers. Such a vortex sheet defines a jet with a top-hat profile. The infinitely thin vortex sheet separates the interior of the jet which flows with axial velocity $u_{j}$ from the surrounding quiescent fluid. While a cylindrical vortex sheet serves as a model for the near field of a jet, a more general velocity profile, such as one derived from similarity analysis, is required to analyse the stability properties of a fully developed jet. Such a similarity solution can be found by considering boundary-layer approximations based on the requirement of a narrow jet (see White 1991). For the fully developed jet profile, Batchelor \& Gill (1962) conclude that the inviscid incompressible uniform-density jet is linearly unstable only to disturbances of azimuthal wavenumber $n=1$ (the so-called helical modes).

A review of jet stability theory (Michalke 1984) catalogued extensions of the inviscid incompressible uniform-density theory which capture various other effects, including 
those of finite Reynolds number and non-zero Mach number. An important aspect of Michalke's paper is the identification of a functional form for the axial velocity profile in the potential core of a jet. This profile is widely used throughout the literature to model the mean flow in the near field of a jet, and is known as 'Michalke's profile two' from the label assigned to it in his paper.

Although compressibility was considered through the Mach-number effect in Michalke's review, another important variable density effect influencing jet stability is that of the jet density ratio $S_{1}$. For upward pointing jets, $S_{1}>1$ yields a positively buoyant jet. This effect is important for variable-density jets, even in the limit of zero Mach number. Thus, the effect of the jet density ratio must be considered separately from the Mach-number effect.

Monkewitz \& Sohn (1988) analysed heated jets for which $S_{1} \neq 1$ by means of an inviscid linear stability analysis. For density ratios above a threshold value of $S_{1}=1.39$, their theory predicted that the variable density jet becomes absolutely unstable. Since common fuels are often less dense than air, this absolute instability may play a key role in the near-field development of fuel jets, and thus is of primary importance to our study.

Monkewitz et al. (1990) further investigated how non-unity density ratios affect jet stability by observing the characteristics of heated jets experimentally. The onset of oscillatory modes in the heated jet experiments was shown to be well correlated to the threshold of absolute instability predicted by the linear stability theory (Monkewitz \& Sohn 1988). Non-unity jet density ratios were also studied for heterogeneous jets by Kyle \& Sreenivasan (1993), who investigated the oscillating modes of helium/air jets.

The role of the air-to-fuel density ratio in jet diffusion flames in normal gravity was studied by Demare \& Baillot (2001). Using laser tomography and particle image velocimetry (PIV), they examined lifted methane flames with an air-to-fuel ratio of about two, which places these jets past the threshold necessary for the onset of selfsustained oscillations. The self-sustained oscillations manifest themselves as a train of ring vortices, which provide one mechanism by which some air is entrained into the middle of the fuel stream. They observed, however, the largest amount of mixing to be caused by axially aligned counter-rotating axial vortex pairs, which remain quasi-steady and draw fuel out of the centre of the jet in a star-shaped pattern. At the base of the lifted flame, these well-organized vortex filaments are able to oppose flame-front propagation, and shape the flame in this region into lobes. The cause of these vortices is thought to be a secondary three-dimensional instability acting upon the initial vortex rings.

In addition to laboratory experiments, a number of numerical simulations of reacting variable-density jets have been performed. Kaplan, Oran \& Baek (1994) performed axisymmetric simulations of lifted methane/air diffusion flames, in which the nozzle exit velocity is sufficient for the flame to detach from the nozzle and stabilize at a distance downstream. In such a configuration, the 'pre-mixing zone' between the nozzle and the flame is exactly a variable-density jet, the perturbation of which can have dramatic consequences for the flame just downstream. Kaplan et al. (1994) observed that the lifted flame base interacts strongly with the KelvinHelmholtz vortical structures which form in the region of the jet shear layer. This gives further justification for considering only non-reacting variable-density jets.

One important difference between the simulations of Kaplan et al. (1994) and the simulations presented in this paper is that the jet in the simulations of Kaplan et al. (1994) had a substantial coflow of air surrounding it. Because of this, a free-slip lateral boundary condition can be employed without the danger of recirculation. In 
this case, all the air entrained into the jet must come from the coflow stream crossing the inlet boundary. The jet considered in this paper, on the other hand, has no coflow, and thus must entrain all of its air across the lateral boundary. Therefore, even a slight amount of coflow is enough to drastically alter the streamlines in the near field of a jet. Also, as Kaplan et al. (1994) point out, while an axisymmetric simulation can provide insight into the initial primary instability of the jet, it cannot capture the fully three-dimensional nature of transition to turbulence.

Mizobuchi et al. (2002) performed a three-dimensional direct numerical simulation (DNS) of a turbulent lifted hydrogen/air flame. Using the DNS data, they identified three components of a lifted hydrogen flame: a leading-edge flame, an inner premixed flame, and outer islands of diffusion flames. They also found that the leading-edge flame, while in the general shape of a ring, has a complicated three-dimensional structure. They found this ring to be unstable with respect to long time scales, which agrees with the observations of Kaplan et al. (1994), as well as the results presented in this paper.

\subsection{Our approach}

In this paper, we study non-reacting variable-density jets through a synergy of DNS and linear stability analysis. In the first part of the paper, we examine a series of three-dimensional DNS of variable-density jets. From the rich dataset provided by DNS, we are able to quantify the transition to turbulence with and without gravity by directly measuring vorticity and baroclinic torque. Furthermore, we measure the selfsustaining oscillations predicted by linear theory directly from the DNS of the fully nonlinear problem. In this way, the linear stability analysis presented in the latter half of this paper serves as a confirmation of what we observe in the DNS.

To implement the DNS, we use the low-Mach-number equations (see McMurtry et al. 1986; McMurtry 1987) from which acoustic waves have been filtered out. Therefore, we consider the stability of the low-Mach-number equations in the latter half of this paper. In order to capture the pinch-point phenomenon associated with absolute instability, the spatial stability of low-Mach-number variable-density jets was considered. Using a matrix method, the entire spatial spectrum with all of its components is derived and catalogued, with the purpose of providing a firm foundation on which to base further work on optimization and control.

In order to quantify the importance of buoyancy to the local stability properties of variable-density jets, a comprehensive parametric study is presented based on the spatial stability analysis. In this study, the effects of buoyancy are compared to the effects of density ratio $S_{1}$, Reynolds number $R e$ and shear-layer thickness.

Finally, the aforementioned synergy between DNS and linear stability analysis is taken a step further by using mean profiles measured from DNS as base flows to which to apply the stability analysis. From this, we compute how the local stability properties change as the jet develops in the axial direction. This analysis allows us to determine at which axial location the jet transitions from absolute instability to convective instability.

\section{Mathematical model}

\subsection{Flow configuration}

Figure 1 shows a schematic diagram of a variable-density jet in a cylindrical domain. The cylindrical domain is oriented vertically so that gravity acts with magnitude $g$ in the downward direction. The jet fluid enters at the bottom of the domain through 


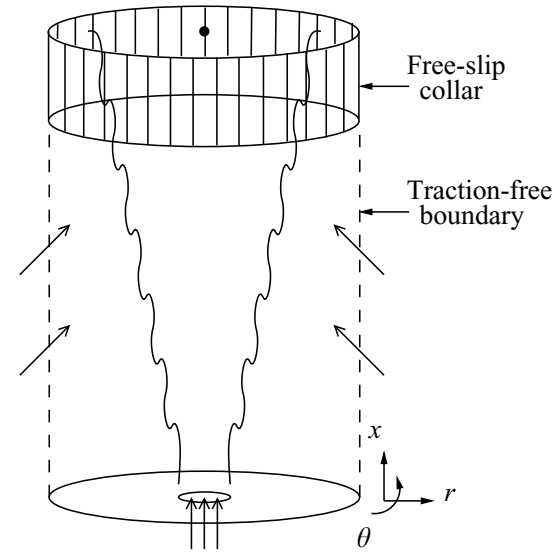

FIGURE 1. Diagram of the computational domain with a traction-free lateral boundary condition and a free-slip collar around the outlet.

a circular orifice of diameter $d$ with maximum velocity $u_{j}$. As the jet develops downstream, ambient fluid is entrained into the flow and mixes with the jet fluid.

An important feature of this flow configuration is the absence of coflow. The circular fuel nozzle is set flush into a flat wall which prohibits flow across the bottom boundary except within the confines of the nozzle. Instead, ambient fluid is allowed to be entrained into the jet flow across the lateral boundaries.

Open boundaries pose inherent difficulties in simulation, because the fluid behaviour just outside of the computational domain must be modelled. It would thus appear advantageous to restrict the lateral boundaries to being closed in the same manner as the bottom boundary. Through numerical experimentation, however, Boersma, Brethouwer \& Nieuwstadt (1998) demonstrated that closing the lateral boundary can have an appreciable effect on an otherwise free jet flow. This happens because the mass entrainment into the jet near the nozzle creates a recirculation zone surrounding the jet. Also, Jendoubi \& Strykowski (1994) point out that counterflow can have a dramatic effect on the stability properties of the jet. Therefore, to minimize the influence of the lateral boundaries on the jet flow, an open-boundary condition must be selected. For our simulations, we have chosen a viscous traction-free boundary condition similar to that implemented by Boersma et al. (1998).

Likewise, the outlet boundary must also be open to allow the jet flow to exit the domain. The goal of the open outlet boundary condition is to model a jet flow into a large ambient space. For this purpose, a convective outflow condition is chosen.

From numerical experiments, it was found that the use of open boundaries for both the lateral and outflow boundaries can lead to inconsistency at the point of intersection of these two boundaries. This inconsistency arises because we have selected different models based on the different types of flow expected at each of these boundaries. Specifically, the viscous traction-free boundary condition best models the small-amplitude irrotational flow across the lateral boundaries, whereas the convective outflow condition captures the large-amplitude highly rotational flow of the jet exiting through the top of the domain.

To avoid computational difficulties associated with the inconsistency of the open boundaries, it was found that the inclusion of a free-slip collar around the outlet greatly improves numerical stability. This collar closes a short section of the lateral boundary immediately adjacent to the outlet boundary, thereby acting as a buffer 
between the two numerical boundary conditions. Unlike the 'dump combustor' case described above, where the entire lateral boundary was closed, a short collar near the outlet does not significantly influence the overall flow. This can be argued by considering streamlines confined to the corner region near the intersection of lateral and outlet boundaries. Without the collar, such streamlines enter the domain across the lateral boundary near the outlet and then quickly exit the domain across the outlet near the lateral boundary. Note that these streamlines do not approach the flow of interest in the centre of the domain, and thus the fluid associated with these streamlines is not entrained into the jet flow. Conversely, most of the fluid which is entrained into the central jet flow has its origin at the lateral boundary, close to the inlet boundary. Therefore, the introduction of a collar in the corner region near the outlet boundary is not expected to influence significantly the jet flow of interest in the centre of the domain.

\subsection{Governing equations}

In the limit of low Mach number, the independent variables of the fully compressible Navier-Stokes equations and the ideal gas law can be represented as power series in $\epsilon=\gamma M a^{2}$, expanded about a condition of zero Mach number Ma. For instance, the pressure $p(\boldsymbol{x}, t)$ can be decomposed into the series

$$
p(\boldsymbol{x}, t)=p^{(0)}(\boldsymbol{x}, t)+\epsilon p^{(1)}(\boldsymbol{x}, t)+\cdots .
$$

Substituting the power series for $p(\boldsymbol{x}, t)$ and for the other independent variables into the governing equations, and equating the coefficients of powers of $\epsilon$ to zero, results in the following lowest-order equations:

$$
\begin{gathered}
\frac{\mathrm{D} \rho}{\mathrm{D} t}+\rho(\nabla \cdot \boldsymbol{u})=0, \\
\nabla p^{(0)}=\mathbf{0}, \\
\frac{\partial \rho \boldsymbol{u}}{\partial t}=-\nabla p^{(1)}+\nabla \cdot\left(\frac{1}{S_{1} R e} \boldsymbol{\tau}-\rho \boldsymbol{u} \boldsymbol{u}\right)-\frac{\rho}{F r^{2}} \hat{\boldsymbol{k}}, \\
\rho \frac{\mathrm{D} T}{\mathrm{D} t}=\frac{1}{S_{1} \operatorname{RePr}} \nabla^{2} T, \\
p^{(0)}=\rho\left[\left(S_{1}-1\right) T+1\right] .
\end{gathered}
$$

With the exception of pressure, only the zeroth-order approximations to the density $\rho$, the velocity $\boldsymbol{u}$, and the reduced temperature $T$ are required in the above lowest-order equations. Therefore, we have dropped the superscript notation, except in the case of pressure, where it is required to distinguish between the zeroth- and first-order approximations.

Because the pressure gradient term in the compressible momentum equation is multiplied by $1 / \gamma M a^{2}$, the first-order pressure gradient, $\nabla p^{(1)}$, balances the other lowest-order variables in $(2.2 c)$. Furthermore, $(2.2 b)$ is obtained by considering that the zeroth pressure gradient term will be much larger than any of the other terms in the compressible momentum equation in the limit of zero Mach number.

Equation $(2.2 b)$ means that $p^{(0)}$ is uniform in space, and, because our problem has open boundaries at which the pressure should remain uniform in time, this means that $p^{(0)}$ will remain uniform in time as well. The term $p^{(0)}$ is sometimes referred to as the thermodynamic or 'slow' component of the pressure - in our case, it does not change at all. On the other hand, the first-order pressure, $p^{(1)}$, is known as the 
hydrodynamic or 'fast' component of the pressure, and adjusts quickly to variations in the flow.

\subsubsection{Non-dimensionalization}

Equation (2.2) has been written in non-dimensional form. Since several possible methods of non-dimensionalization exist, it is important to specify our technique carefully here. In the following, the primes denote dimensional quantities. The primitive variables $\rho, \boldsymbol{u}$ and $p$, space and time are non-dimensionalized as follows:

$$
\rho=\frac{\rho^{\prime}}{\rho_{0}}, \quad \boldsymbol{u}=\frac{\boldsymbol{u}^{\prime}}{u_{j}}, \quad p=\frac{p^{\prime}}{\rho_{0} R T_{0}}, \quad \nabla=d \nabla^{\prime}, \quad t=\frac{t^{\prime} u_{j}}{d},
$$

where $\rho_{0}, T_{0}, u_{j}$ and $d$ represent ambient density, ambient temperature, the maximum fluid velocity at the nozzle and the nozzle diameter, respectively. Note that nondimensionalizing pressure in the above manner results in a Mach number squared multiplying the pressure gradient term in the compressible momentum equation which allows the low-Mach-number equations to be expressed in the form presented in (2.2).

The non-dimensional viscous stress tensor $\boldsymbol{\tau}$ and acceleration due to gravity $\boldsymbol{g}$ are defined as follows:

$$
\boldsymbol{\tau}=\frac{\boldsymbol{\tau}^{\prime} d}{\mu u_{j}}, \quad \boldsymbol{g}=\frac{\boldsymbol{g}^{\prime}}{g}=-\hat{\boldsymbol{k}}
$$

where $g$ is the magnitude of acceleration due to gravity, and $\mu$ is the fluid viscosity which is taken to be constant throughout the flow. In our study, we neglect variable viscosity effects, as they might mask other, more fundamental effects. Notice also that $\boldsymbol{g}^{\prime}$ is simply the unit vector in the direction in which gravity acts.

Finally, the temperature field is scaled by the ambient temperature $T_{0}$ and the maximum temperature $T_{\max }$,

$$
T=\frac{T^{\prime}-T_{0}}{T_{\max }-T_{0}}
$$

so that the non-dimensional temperature $T$ varies between zero and unity.

The specific choice of scales for the non-dimensionalization of the governing equations yields a set of key parameters that determine the flow dynamics and stability behaviour. This set includes the Reynolds number $R e$, the Froude number $\mathrm{Fr}$, the density ratio $S_{1}$, and the Prandtl number $\mathrm{Pr}$, as written below.

$$
R e=\frac{\rho_{j} u_{j} d}{\mu}, \quad F r=\frac{u_{j}}{\sqrt{g d}}, \quad S_{1}=\frac{\rho_{0}}{\rho_{j}}=\frac{T_{\max }-T_{0}}{T_{0}}+1, \quad \operatorname{Pr}=\frac{\mu_{0} c_{p}}{\lambda} .
$$

Here, $T_{\max }$ is the temperature at the jet exit, and the Reynolds number is defined in terms of the density of the jet fluid, $\rho_{j}$. We point out that the kinematic viscosity $v=\mu / \rho_{j}$ changes as the density changes; thus, as the jet density decreases, the jet velocity must increase to yield a constant Reynolds number.

The Froude number provides information about the magnitude of buoyant forces compared to inertial forces. As the Froude number increases, buoyant forces become less important compared to inertial and viscous forces. As the condition of zero gravity $(g \rightarrow 0)$ is approached, the Froude number becomes infinitely large $(\mathrm{Fr} \rightarrow \infty)$, and the buoyant term vanishes altogether.

The energy equation $(2.2 d)$ containing the Prandtl number was derived by assuming that the specific heat $c_{p}$ and the thermal conductivity $\lambda$ are constant throughout the fluid, and that the Mach number is low enough that only the advective and diffusive terms remain. For a detailed derivation of this equation, see Poinsot \& Veynante 
(2001) and Bird, Stewart \& Lightfoot (1960). For computational efficiency, the nondimensional form of the ideal gas law $(2.2 e)$ can be used to eliminate $T$ in $(2.2 d)$, so that

$$
\frac{\mathrm{D}}{\mathrm{D} t}\left(\frac{1}{\rho}\right)=\frac{1}{\rho} \frac{1}{S_{1} \operatorname{RePr}} \nabla^{2}\left(\frac{1}{\rho}\right),
$$

assuming, without loss of generality, that $p^{(0)}=1$.

\section{Numerical simulation}

Direct numerical simulations have been performed to investigate the disturbance dynamics in variable-density jets. The results of these simulations are then used to motivate and guide computational stability analyses. The goal is to extract a physical understanding of the phenomena observed in the direct numerical simulations.

\subsection{Numerical methods}

To implement the DNS, a computer code was developed to solve the low-Machnumber equations (2.2) in cylindrical coordinates on a staggered mesh. In order to resolve as large a range of scales as possible, highly accurate sixth-order compact schemes are used to compute spatial derivatives in the non-periodic directions $(r$ and $x$ ) (see Lele 1992). In the remaining periodic direction $(\theta)$, an even more accurate spectral method is employed. Also, using a staggered mesh has the added benefit of being able to treat the advection of high wavenumbers more accurately than is possible on a collocated mesh (see Pierce \& Moin 2001).

To complement the accuracy of the space differencing schemes, third-order AdamsBashforth time differencing (see Durran 1999) is used to advance the advection and diffusion terms in the momentum and energy equations. The effect of the pressure gradient is then treated with a projection method which enforces the constraint imposed by the continuity equation (see Chorin 1967).

The low-Mach-number equations differ from the anelastic equations sometimes used in atmospheric science by including the term $\partial \rho / \partial t$ in the continuity equation (2.2a), which allows for the advection of fluid pockets of varying density. McMurtry (1987) points out, however, that any slight error in the calculation of $\partial \rho / \partial t$ can have far-reaching consequences owing to the elliptic nature of the constraint imposed by the continuity equation. Therefore, in order to compute this term as accurately as possible, a predictor-corrector scheme is used, similar to the type employed by Najm, Wyckoff \& Knio (1998). Najm et al. (1998) report handling density ratios as large as $10: 1$, although in the present work, a maximum density ratio of $4: 1$ is considered.

Finally, an asymptotic method is used to treat the centreline, since the governing equations in cylindrical coordinates contain an apparent singularity at $r=0$ (see Constantinescu \& Lele 2002). Furthermore, in the region near to the centreline, a spectral cutoff filter is used to avoid numerical difficulties associated with azimuthal clustering of grid points.

For further details about the numerical method, refer to Nichols (2005).

\subsection{Simulation configurations}

In order to study the effects of buoyancy on the stability of variable-density jets, we present two simulations with identical configurations except for one flow parameter: the Froude number. In one case, the Froude number was specified to be infinite $(F r=\infty)$ corresponding to a non-buoyant jet in zero gravity. In the other simulation, the Froude number was set to $F r=8$, corresponding to a buoyant jet. The simulations 


$\begin{array}{ccccccccc}\text { Simulation } & S_{1} & R e & F r & \frac{R_{u}}{\theta_{u}} & \frac{R_{\rho}}{\theta_{\rho}} & \operatorname{Pr} & S c & \text { Perturbation level } \\ \text { A2 } & 4.0 & 1000.0 & \infty & 12.5 & 12.5 & 0.7 & 0.7 & 5 \% \\ \text { A3 } & 4.0 & 1000.0 & 8.0 & 12.5 & 12.5 & 0.7 & 0.7 & 5 \%\end{array}$

TABLE 1. Variable density jet simulation runs.

are labelled A2 and A3, respectively, in order to correspond to the notation of Nichols (2005).

In order to simulate a jet in the transitioning regime, a Reynolds number of $R e=1000$ was selected for both simulations. The Prandtl number, Pr, and Schmidt number, $S c$, were both set to 0.7 , implying a unity Lewis number condition. The flow parameters for both simulations have been summarized in table 1 .

The simulations used a computational domain of radial extent $R=4$ and axial extent $X=18$ with a discretization resolution of $128 \times 512 \times 256$ grid points in the $r, x$ and $\theta$ directions, respectively. Because of the non-dimensionalization introduced in $\S 2$, the distances $R$ and $X$ are both measured in terms of the nozzle diameter.

The same inlet velocity and density profiles were used for both simulations. The inlet axial velocity profile chosen corresponds to Michalke's profile number two (see Michalke 1984), with a radius $R_{u}=d / 2=0.5$ and a radius-to-momentum thickness ratio $R_{u} / \theta_{u}=12.5$ :

$$
u_{x}(x=0, r)=\frac{1}{2}\left(1+\tanh \left(\frac{1}{4} \frac{R_{u}}{\theta_{u}}\left(\frac{R_{u}}{r}-\frac{r}{R_{u}}\right)\right)\right) .
$$

The inlet density profile was also assumed to take on the functional form given by Michalke's profile two, but subtracted from 1 so that the density is lowered in the centre of the jet. Expressed mathematically, the density profile at the inlet is given by the following formula:

$$
\rho(x=0, r)=1-\left(1-\frac{1}{S_{1}}\right) \frac{1}{2}\left(1+\tanh \left(\frac{1}{4} \frac{R_{\rho}}{\theta_{\rho}}\left(\frac{R_{\rho}}{r}-\frac{r}{R_{\rho}}\right)\right)\right),
$$

with $R_{\rho} / \theta_{\rho}=12.5$. In a following section, we will investigate how $R_{u} / \theta_{u}$ and $R_{\rho} / \theta_{\rho}$ affect the jet stability properties. These parameters have been written in such a way that higher values correspond to thinner shear layers, and, as we will see, more unstable jets.

As shown in figure 2, the ambient-to-jet density ratio was chosen to be four $\left(S_{1}=4.0\right)$, yielding a jet which is four times less dense in the centre than at the edges. This density ratio was chosen to ensure that the jet is well within the absolutely unstable regime, according to Monkewitz \& Sohn (1988). While an oxidizer-tofuel density ratio of $S_{1}=4.0$ might be realizable in some specialized combustion applications, it should be noted that for methane flames $S_{1}$ is closer to 2.0. This means that methane flames are closer to the boundary between the convective and absolute instability regimes, but, as we will show, $S_{1}=2.0$ still yields an absolutely unstable jet. The advantage of choosing a larger density ratio is that the effects of the instability are somewhat stronger and so reach a (statistically) steady state more quickly, allowing for shorter computer simulation runs.

The flow is initially chosen to be parallel throughout the domain with no axial variation allowed in any of the flow variables. In order to break the symmetry, random 


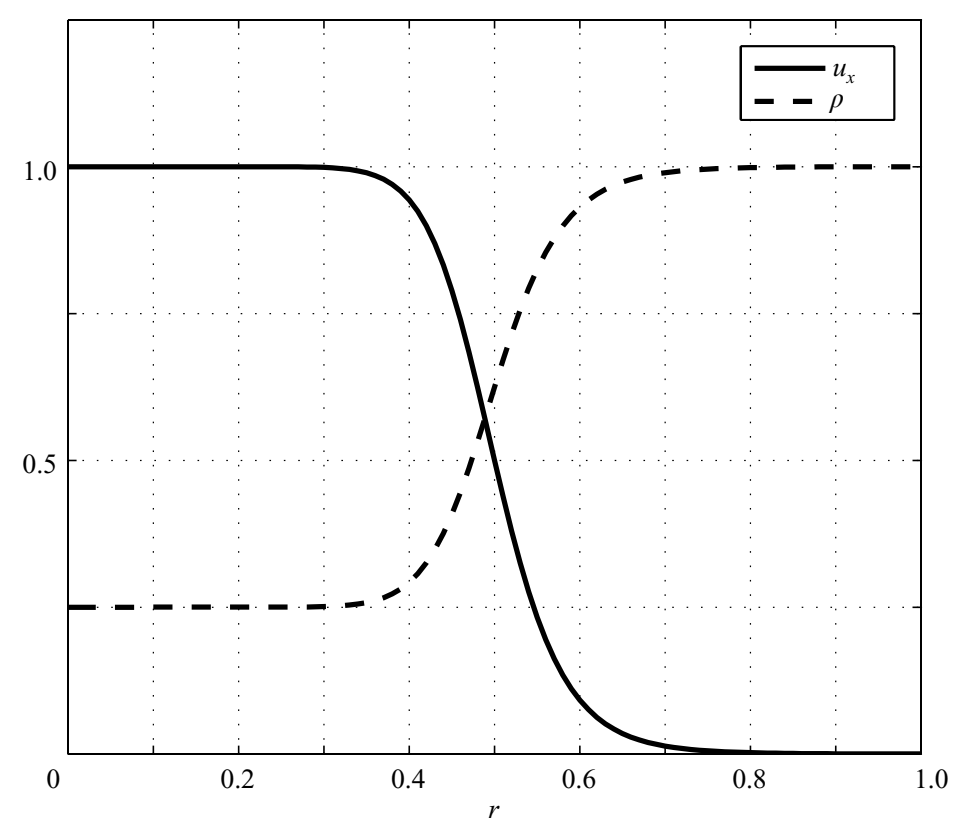

FIGURE 2. Inlet profiles for variable-density-jet simulations.

perturbations were added as white noise to the initial velocity field in the vicinity of the parallel flow shear layer. The maximum amplitude of these perturbations was taken to be $5 \%$ of the peak jet inlet velocity, or $0.05 u_{j}$. The addition of perturbations to the flow can be thought of as a type of initial low-level forcing, after which the flow is left to develop purely in accordance to the governing equations (2.2). In other words, random perturbations are added only at time $t=0$ and at no other times, so fluctuations present in the flow at later times are a direct result of the initial perturbations. This represents a more controlled environment than found in the laboratory where random perturbations can occur at any time.

Perturbing the initial flow is necessary to allow the flow to develop three-dimensional features, because without some initial perturbation to the system, the numerical scheme would constrain the flow to remain axisymmetric, possibly missing important physical processes. As we will see, the absolute instability which develops in the near field of the flow is observed to be axisymmetric, i.e. of azimuthal wavenumber $n=0$. Perturbations of other azimuthal wavenumbers either damp out or grow only as they are convecting downstream. Because of this, the perturbations added to the initial flow field do not affect the very near field of the jet in the limit of $t \rightarrow \infty$, except by triggering a large-amplitude, rather robust, axisymmetric absolute instability. It should be noted that the addition of three-dimensional perturbations is not completely necessary to trigger this absolute instability, because just the initial axisymmetric adjustment from parallel flow to that of a naturally spreading jet in the absence of perturbations was found to be sufficient to trigger this instability. On the other hand, as the initial three-dimensional perturbations convect downstream, some will saturate and cause the flow to transition to a fully three-dimensional turbulent flow. Worse, without three-dimensional perturbations to introduce non-zero values to azimuthal wavenumbers $n>0$, the numerical method would constrain these wavenumbers to remain exactly equal to zero throughout the simulation. In this sense, 
the initial perturbations are critical to this study, since turbulence cannot develop in a flow that is constrained to be axisymmetric.

\subsection{Direct numerical simulation results}

Figures 3 and 4 show the time development of the jet from initial conditions for the two different Froude numbers, $F r=\infty$ (the non-buoyant case) and $F r=8.0$ (the buoyant case). Each successive image is separated from the previous one by a nondimensional time of 12 , so that a non-dimensional timespan of 72 is considered in each case. With a time step of $\Delta t=0.004$, this corresponds to 18000 time steps. Part $(h)$ in each sequence does not represent a new time, but rather displays an axial cross-section of the domain at the final time step shown in the seventh image.

In order to visualize the flow, a conserved scalar field $Z$ was carried through the computation. The inlet boundary condition $Z(x=0, r)$ was set to the same functional form as given on the right-hand side of (3.1), so that $Z=1$ at the centre of the nozzle and $Z=0$ in the ambient regions. The term $Z$ is often referred to as the mixture fraction of jet fluid (fuel) and ambient fluid (air). The blue surfaces in these plots are in each case the isosurface $Z=0.111$. If $Z=0.111$ represents the ideal mixture of fuel and air required for combustion, then this surface is referred to as the stoichiometric surface.

In both cases, the flow initially adjusts to become non-parallel. By non-dimensional time $t=48$ (figures $3(e)$ and $4(e)$ ), however, the initial adjustment has left the computational domain. In the final images of both sequences, we observe that the laminar inflow at the bottom of the domain enters first into an oscillatory state which is dominated by a train of Kelvin-Helmholtz-type vortices convecting downstream along the shear layer. The roll-up of the Kelvin-Helmholtz vortices is visualized in an axial cross-section in part $(h)$ of each sequence.

In the case of the non-buoyant jet, long axially-aligned streak-like structures are observed to develop in figures $3(e-g)$ and $4(e-g)$ in the region $4<x<10$. These structures are sometimes referred to as side jets because they form by jet fluid being ejected from the jet centre in a star shaped pattern seen in a radial cross-section in figure 5(a). From animations of this flow (not shown) the side jets were observed to maintain their azimuthal position throughout many cycles of the oscillation associated with the passage of the Kelvin-Helmholtz rings. The side-jet formation is thought to be a result of a secondary instability, the analysis of which is beyond the scope of the present discussion. However, side-jet formation is markedly absent in the case of the buoyant jet, the result of which is that the transition to the quasi-turbulent flow observed at the top of both domains is much more abrupt in the case of the buoyant jet. This is consistent with the findings of Bahadori et al. (1992) who observed buoyant jet flame instabilities forming at the flame tip and then gradually incorporating the entire flame as flow rates were increased, whereas microgravity flame instabilities first appeared throughout the entire flame.

\subsection{Vorticity dynamics}

In order to quantify the difference in abruptness of transition observed in the previous section, we consider vorticity dynamics. The vorticity equation for a variable density flow can be written as:

$$
\frac{\mathrm{D}}{\mathrm{D} t}\left(\frac{\boldsymbol{\omega}}{\rho}\right)=\left(\frac{\omega}{\rho}\right) \cdot \nabla \boldsymbol{u}+\frac{\nabla \rho \times \nabla p}{\rho^{3}}+\text { diffusive terms. }
$$

In this equation, the fluid vorticity is denoted by $\boldsymbol{\omega}=\nabla \times \boldsymbol{u}$. The left-hand side 

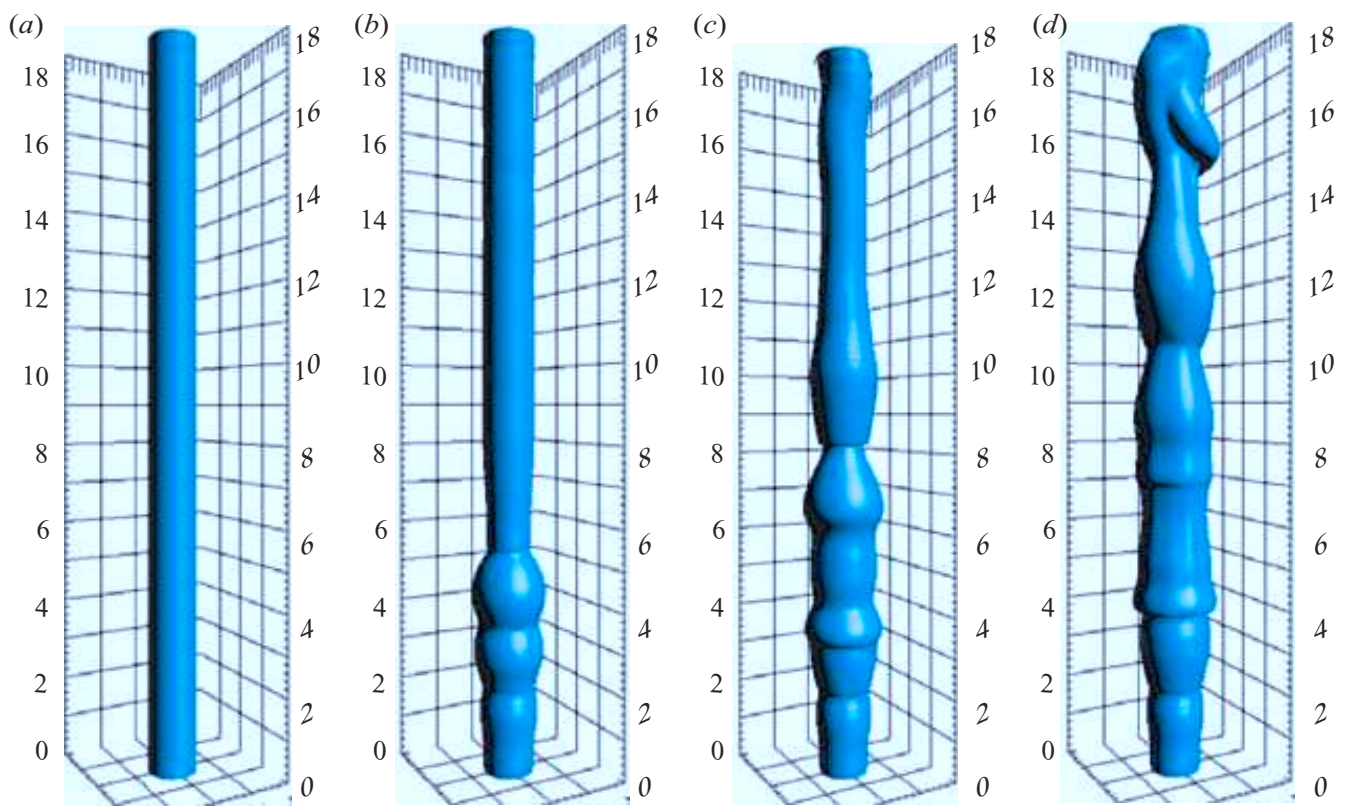

(e)
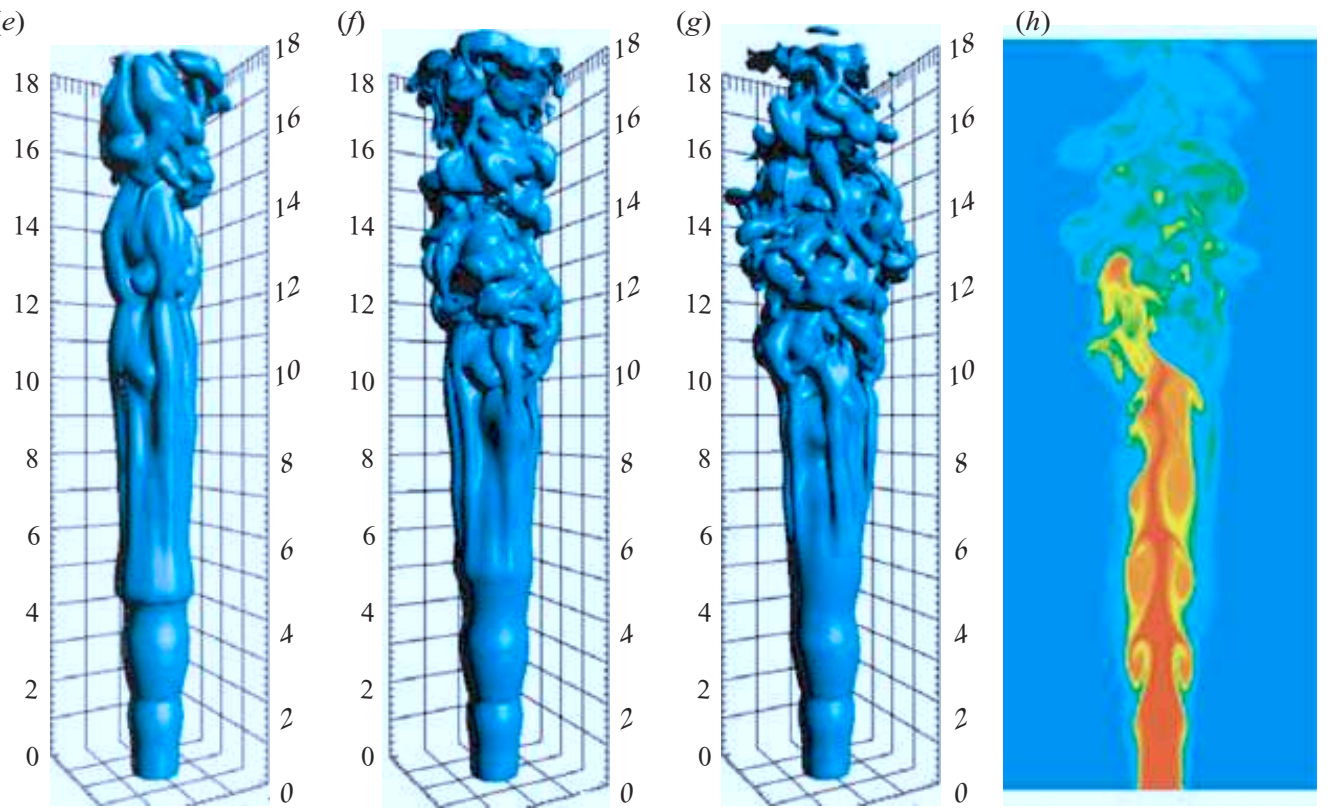

Figure 3. Simulation A2 $(F r=\infty)$ : time series of the (stoichiometric) isosurface $Z_{s t}=0.111$. A non-dimensional time of 12.0 elapsed between successive images. $(h)$ Contours of $Z$ on an axial cross-section taken through $(g)$.

represents the material derivative of the weighted vorticity $\omega / \rho$, which is balanced on the right-hand side by first the vortex stretching/tilting term, then the baroclinic torque and, finally, the diffusive terms. Note that in this moderate Reynolds-number flow, the diffusive terms are relatively small. Moreover, the diffusive terms usually act to lessen gradients of vorticity instead of sharpening them, as is possible with the 

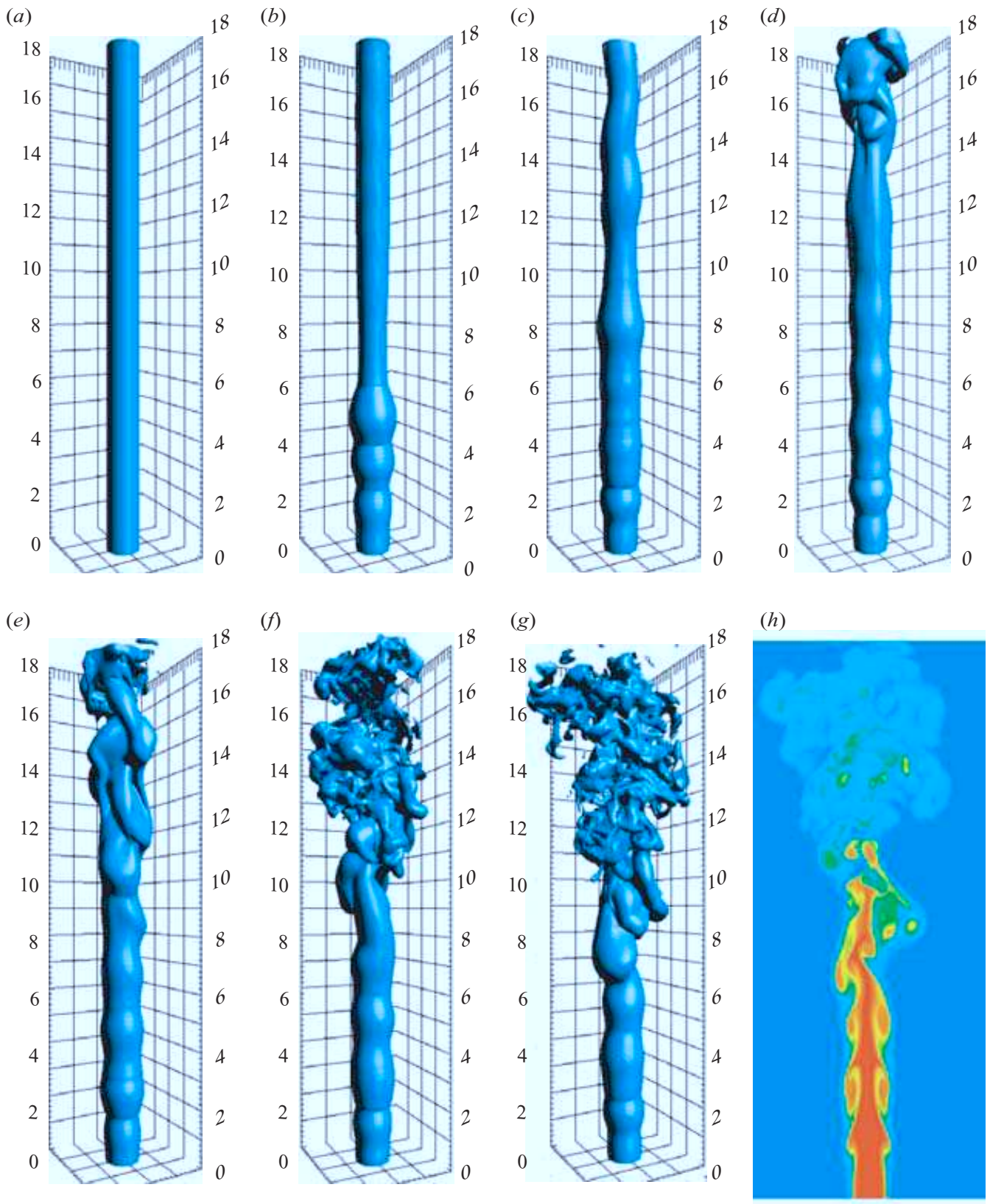

Figure 4. Simulation A3 $(F r=8.0)$. Details as for figure 3.

vortex stretching and baroclinic torque terms. Also, by writing this equation as the transport of the weighted vorticity $\omega / \rho$ rather than just the simple vorticity $\omega$, we eliminate the need for a dilatation term.

To measure the increasing three-dimensionality of the vorticity as the jet transitions to turbulence, we can compute the 'weighted' root mean square (r.m.s.) over radial cross-sections of each of the three vorticity components. For instance, for a given axial location the weighted r.m.s. value of the $\theta$ component of vorticity can be calculated 


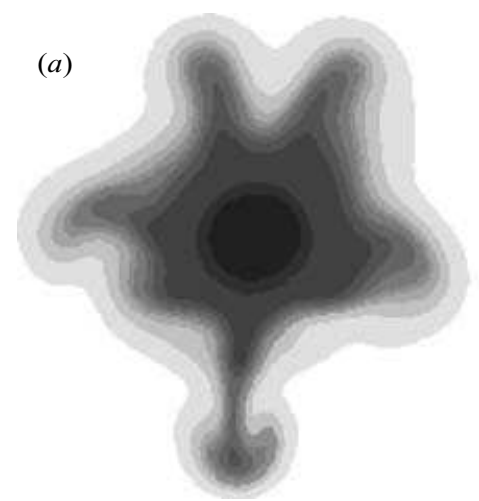

(b)

Figure 5. Contours of $Z$ on radial cross-sections taken at $x=8$ and $t=72$ (corresponding to figures $3(\mathrm{~g})$ and $4(\mathrm{~g}))$ of $(a)$ the non-buoyant jet and $(b)$ the buoyant jet. Side jet formation is observed only in the non-buoyant case.
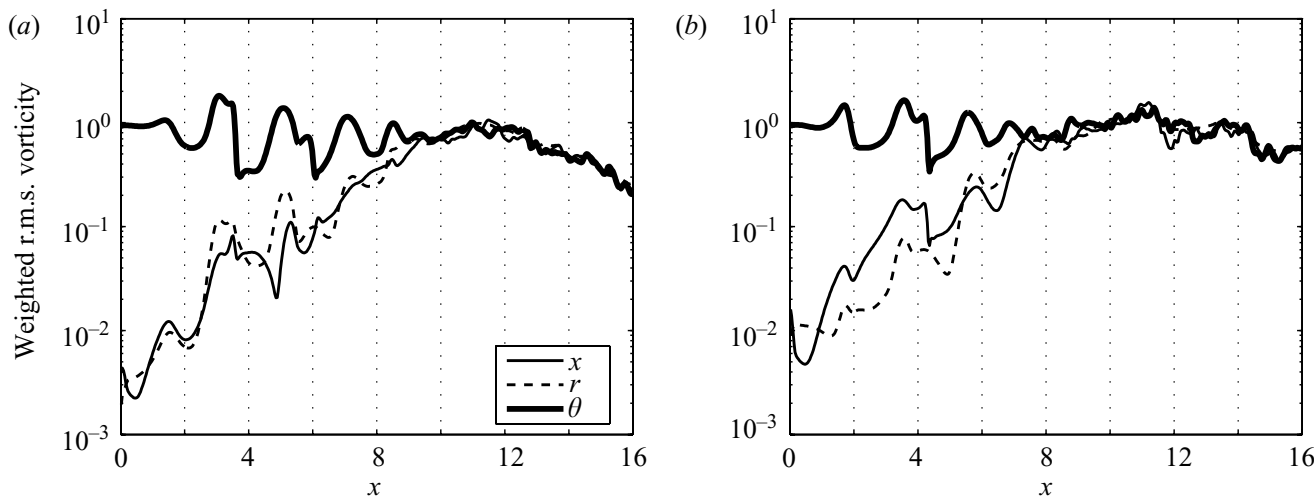

FIGURE 6. The spatial development of vorticity in $(a)$ a non-buoyant and $(b)$ a buoyant jet, taken at non-dimensional time $t=72.0$.

as:

$$
\left(\frac{\omega_{\theta}}{\rho}\right)_{r m s}=\left(\frac{1}{A} \iint\left(\frac{\omega_{\theta}}{\rho}\right)^{2} r \mathrm{~d} r \mathrm{~d} \theta\right)^{1 / 2},
$$

where $A$ is the cross-sectional area.

The weighted r.m.s. values of the three vorticity components are plotted as functions of $x$ in figure 6 . The figure shows that for both the non-buoyant and buoyant jet, the jet enters the domain at $x=0$ containing only azimuthal vorticity. Also, for both cases, the other components of vorticity begin to grow, beginning at about axial location $x=6$. For the non-buoyant jet, these other components of vorticity reach the same magnitude as the azimuthal vorticity at approximately $x=9.5$, after which the three components of vorticity are comparable in magnitude, indicating a region of turbulence before the flow leaves the domain. For the non-buoyant jet, the long region of exponential growth of the radial and axial vorticity is thought to be due to the presence of a secondary instability, which has the effect of stretching and tilting the vorticity (see Brancher, Chomaz \& Huerre 1994). In the case of the buoyant jet, however, this transition happens more abruptly, with the growing components of vorticity reaching the same magnitude as azimuthal vorticity at axial location 

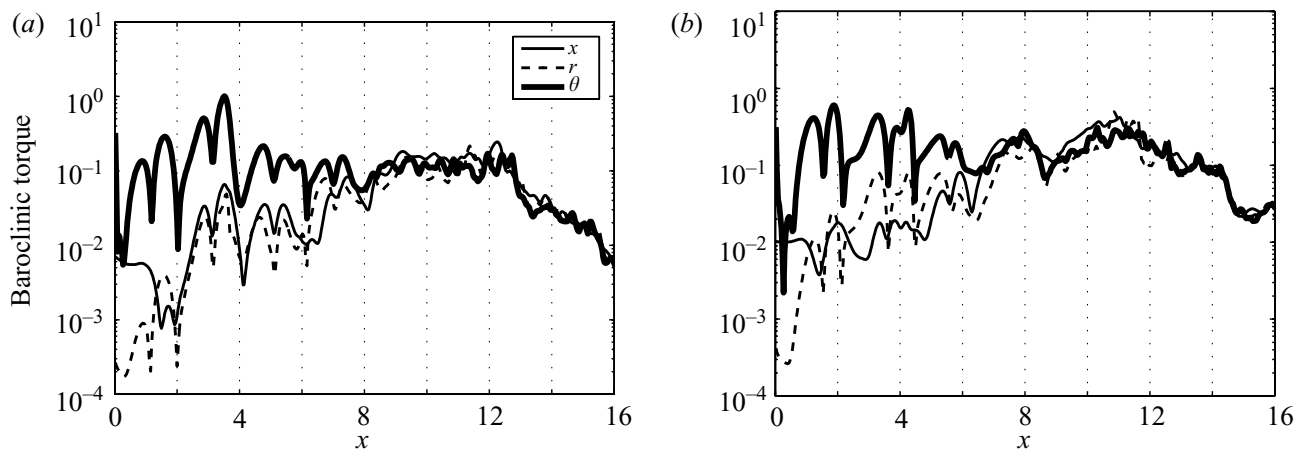

Figure 7. The spatial development of baroclinic torque in $(a)$ a non-buoyant and $(b)$ a buoyant jet, taken at non-dimensional time $t=72.0$.

$x=7.5$. This trend is confirmed by a simulation (not shown) of a jet in strong gravity $(F r=4)$ in which the increased buoyancy produces an even more abrupt transition (see Nichols 2005).

The pulsations evident in the weighted r.m.s. azimuthal vorticity curve in figure 6 are due simply to the roll-up of ring vortices. Since we are integrating over a crosssection in (3.4), cross-sections intersecting a vortex ring will naturally have a larger value of $\left(\omega_{\theta} / \rho\right)_{r m s}$ than those cross-sections that do not. In other words, the primary instability has the effect of concentrating azimuthal vorticity into spatially coherent flow structures (the vortex rings). Note that these oscillations are evident well into the region of secondary instability and even to some extent in the turbulent region. From this, we can conclude that the primary instability, which can be explained by linear theory, has a significant effect on the overall flow development.

The effect of the moderate Reynolds number, $R e=1000$, is evident in figure 6 . For higher Reynolds numbers, we would expect all three vorticity components to increase as we move downstream in the turbulent region, owing to the high amount of vortex stretching and tilting exhibited by turbulent flows. However, in our flow, the three components show a gradual decrease because the vorticity is diffused by viscosity. Therefore, it might be better to call this region the quasi-turbulent region, since it exhibits some characteristics of turbulence (approximate isotropy of vorticity), but not others.

In the turbulent region, the total level of vorticity is greater in the case of the buoyant jet than in the case of the non-buoyant jet. As one might guess, this increased level of vorticity in the turbulent region of buoyant jets can in part be attributed to increased baroclinic torque acting as a source for vorticity. Figure 7 shows the axial development of the r.m.s. components of baroclinic torque, computed using the same type of spatial averaging as in (3.4). In much the same manner as the vorticity, the baroclinic torque is initially directed only in the azimuthal direction in the region near to the inlet, after which the three components of the torque equalize as the jet develops downstream. From our simulations, we observe that the magnitude of the baroclinic torque in the quasi-turbulent region is indeed nearly twice as large in the buoyant jet than in the non-buoyant jet.

Buoyancy seems to have the opposite effect in the region of primary instability. Note the four large peaks in the $\theta$ curves between $x=0$ and $x=4$ in figure 7 . The amplitude of the second and fourth peaks is substantially reduced in the case of the buoyant jet. The origin of these peaks can be explained by considering a 


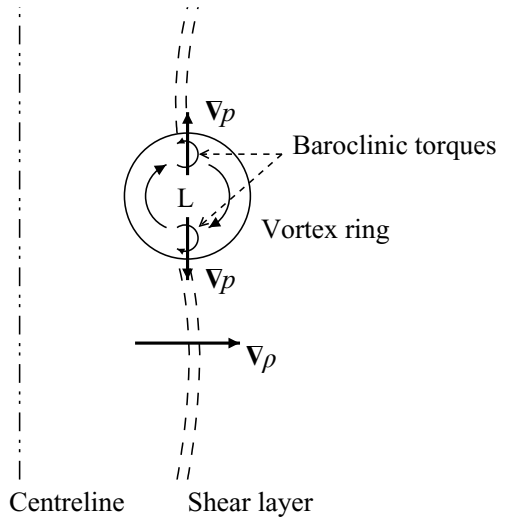

FIGURE 8. Schematic of baroclinic torque in vortex rings.

schematic of an axial cross-section of a vortex ring (figure 8). There is a region of low pressure associated with the centre of the vortex ring, which produces oppositely directed pressure gradients at the top and bottom of the vortex, as shown by the two vertical arrows labelled $\nabla p$. In general, the density gradient vector, shown as the horizontal arrow labelled $\nabla \rho$, is directed away from the centreline. The misalignment of the pressure and density gradients produces baroclinic torques in the top half and the bottom half of the vortex as shown by the small arcs. In the bottom half of the vortex ring, the baroclinic torque tends to be in the same direction as the rotation of the vortex ring itself, whereas the baroclinic torque in the upper half of the vortex opposes the rotation of the vortex.

In figure 7 , the four peaks in $\theta$ torque are due to two vortex rings. The first and third peaks are due to the baroclinic torque in the bottom halves of these rings and the second and fourth peaks are due to the oppositely directed torque in the top half of the rings. Recall that we are plotting r.m.s. quantities in these figures, so information about the sign of the torques is lost upon squaring.

In figure 8 , we have assumed a condition of zero gravity. When gravity is added, however, a mean hydrostatic pressure gradient develops which would be directed downwards in the figure. This mean gradient has the effect of weakening the upwards directed pressure gradient in the top half of the vortex ring and strengthening the downwards directed pressure gradient. This causes a corresponding weakening of baroclinic torque in the upper half of the vortex ring and a strengthening of the torque in the bottom half of the ring. Since the second and fourth peaks of $\theta$ torque in figure 7 correspond to baroclinic torques in the upper half of the rings, this explains why these peaks are diminished going from the non-buoyant jet to the buoyant jet. As expected, the first peak in figure 7 decreases going from the non-buoyant jet to the buoyant jet. The third peak decreases slightly, however, which does not fit the pattern, but note that it is spread over a larger axial distance. This decrease might then be explained by considering that the acceleration of the centreline velocity produced by buoyancy counteracts the expected increase in torque in the bottom half of the vortex rings by spreading the pressure gradient over larger axial distances.

\subsection{System response}

In order to capture the frequency response of the system, time histories of the radial velocity were recorded at stations located at different distances downstream of the shear layer. The stations were all located at the same radial coordinate of $r=0.5$, which 


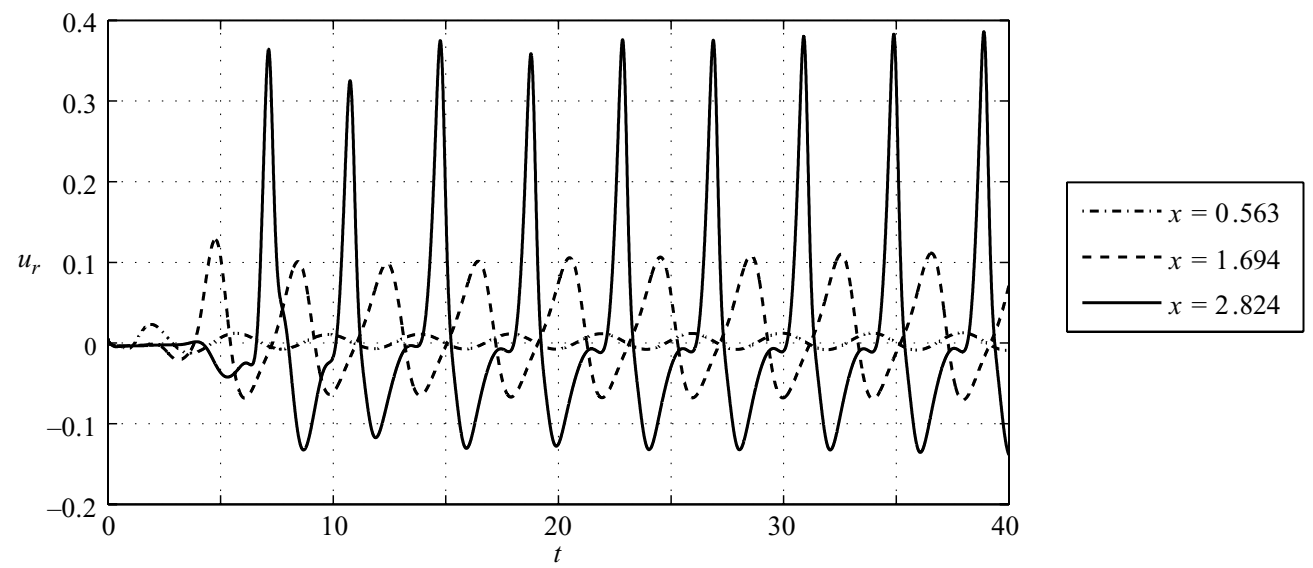

FIGURE 9. Time histories of radial velocity in region of primary instability for zero gravity jet (simulation A2).

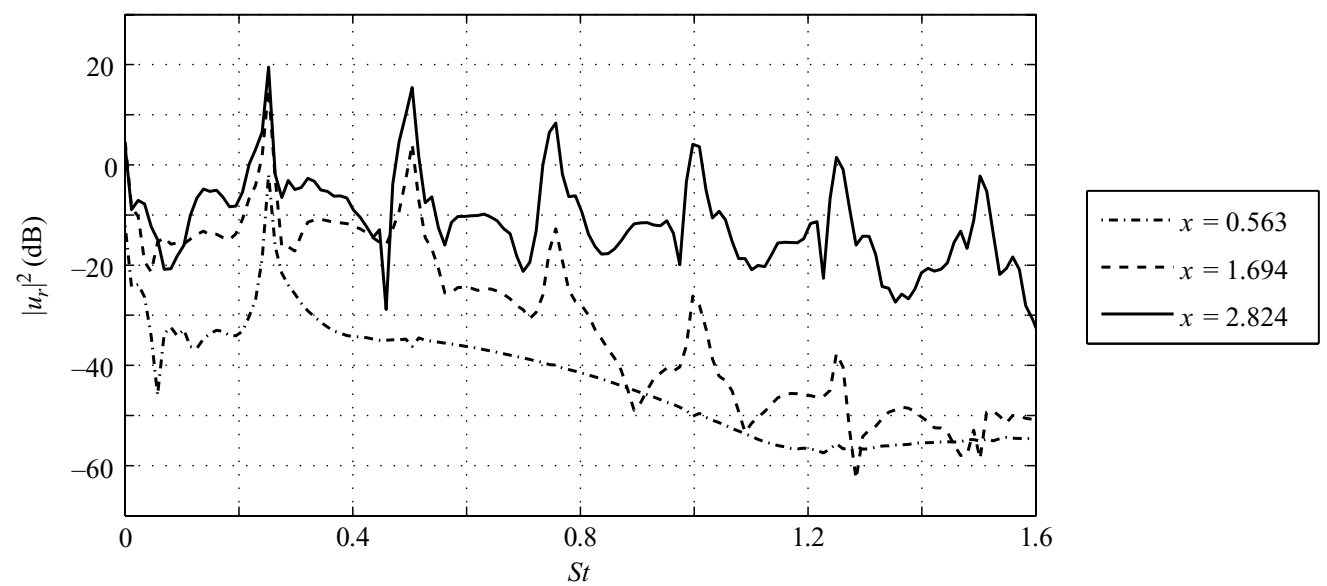

FIGURE 10. Power spectra of radial velocity signal in region of primary instability for zero gravity jet (simulation A2).

placed them at the centre of the shear layer in the parallel flow used for the initial conditions. Also, the azimuthal coordinate of each station was fixed at $\theta=0$. Figure 9 plots histories from three stations, all of which were located within the region of primary instability. These data were generated by simulation A2 of a jet in zero gravity.

Figure 9 captures the near-field behaviour of the jet, which is dominated by a self-sustaining mode-locked oscillation with a Strouhal number, $S t$, of:

$$
S t=\frac{f d}{u_{j}}=0.250,
$$

where $f=1 / T$ and $T$ is the non-dimensional period for one complete cycle. This oscillation is due to the primary instability of the jet.

Another way to interpret the development of the jet in the region of primary instability is to consider the power spectra of the signals of figure 9. These spectra are plotted in figure 10. Note that the spectrum for the station closest to the inlet is extremely narrow-banded, being almost entirely comprised of a pure 


$\begin{array}{ccr}\text { Simulation } & F r & S t \\ \text { A2 } & \infty & 0.250 \\ \text { A3 } & 8.0 & 0.270\end{array}$

TABLE 2. Measured frequency of primary instability.

fundamental oscillation at the aforementioned Strouhal number. Because of inherent nonlinearities, harmonics appear as the jet develops downstream, and signals measured at downstream stations become increasingly broadbanded. However, the flow at all stations in the region of primary instability is dominated by the fundamental frequency, as can be seen from the figure, taking into account that a logarithmic scale was used for the vertical axis.

Table 2 summarizes the frequencies observed from DNS in the region of primary instability for cases A2 and A3. The $8 \%$ increase in frequency in the buoyant case is believed to be significant because the measurement error of the peak-to-peak period $T$, based on a sample time $\Delta t$, was about $0.1 \%$. Statistical scatter was avoided by averaging ten peak-to-peak measurements of $T$ for ten complete cycles in each case. Furthermore, this trend of increasing frequency with increasing buoyancy is confirmed by a simulation (not shown) of a jet in strong gravity $(F r=4)$ in which an even greater frequency is observed (see Nichols 2005).

\section{Stability analysis}

Since the axial velocity and density profiles of the jet satisfy the inflection point theorem, extended to include variation in the density, it meets the necessary condition for instability according to Rayleigh's inflection-point criterion (see e.g. Drazin \& Reid 1981). Since this is neither sufficient proof of instability nor provides any detail about the observed instability behaviour, we must verify by means of a linear stability analysis that variable-density jets are indeed unstable to low-level perturbations, which will be the primary topic of this section.

\subsection{Linearized equations}

The low-Mach-number equations are linearized by considering small wavelike perturbations of the form

$$
q(x, r, t)=\tilde{q}(\alpha, r, \omega) \exp (\mathrm{i} \alpha x-\mathrm{i} \omega t),
$$

about mean profiles $\bar{u}$ and $\bar{\rho}$ such as those shown in figure 2. Here, $\alpha$ denotes the complex axial wavenumber and $\omega$ denotes the complex frequency. Substituting solutions of this form into the low-Mach-number equations and neglecting terms of higher order in the perturbation quantities, yields a system of Fourier-Laplace transformed equations describing an eigenvalue problem for either $\alpha$ or $\omega$, given that the other is known. For example, if a fixed, real $\alpha$ is specified, then the eigenvalue problem can be solved for complex eigenvalues $\omega_{j}$. This case is known as the temporal problem since the eigenvalues $\omega_{j}$ determine the system's temporal response to perturbations of axial wavenumber $\alpha$.

For the remainder of this paper, however, we will be primarily concerned with the spatial problem in which a real frequency $\omega$ is given and the eigenvalue problem is solved for complex eigenvalues $\alpha_{j}$. By considering the spatial problem, we can examine how jet instabilities grow in space at different frequencies. The spatial 
problem is slightly more difficult than the temporal problem because the eigenvalue $\alpha$ appears nonlinearly in the above system, whereas $\omega$ appears only linearly. This is because the original untransformed equations contained second derivatives in space, but only first derivatives in time. The nonlinear (quadratic) eigenvalue problem can be recast into a linear one, however, by introducing the additional variables $\tilde{u}_{x}, \tilde{v}_{x}$ and $\tilde{\rho}_{x}$, as the following system of equations shows. Similar results can be obtained by using a companion matrix technique.

$$
\begin{aligned}
& \mathrm{i} \alpha \tilde{u}=\tilde{u}_{x} \\
& \mathrm{i} \alpha\left(-\frac{4}{3 S_{1} R e} \tilde{u}_{x}+\tilde{p}\right)=\left.\mathrm{i} \omega \bar{\rho}+\frac{1}{S_{1} \operatorname{Re}}\left(\frac{\partial^{2}}{\partial r^{2}}+\frac{1}{r} \frac{\partial}{\partial r}\right)\right) \tilde{u}-\bar{\rho} \bar{u} \tilde{u}_{x}-\bar{\rho} \bar{u}^{\prime} \tilde{v} \\
&+\frac{1}{3 S_{1} \operatorname{Re}}\left(\frac{\partial}{\partial r}+\frac{1}{r}\right) \tilde{v}_{x}-\frac{1}{F r^{2}} \tilde{\rho} \\
& \mathrm{i} \alpha \tilde{v}=\tilde{v}_{x} \\
& \mathrm{i} \alpha\left(-\frac{1}{S_{1} \operatorname{Re}} \tilde{v}_{x}\right)=\frac{1}{3 S_{1} \operatorname{Re}} \frac{\partial \tilde{u}_{x}}{\partial r}+\left[\mathrm{i} \omega \bar{\rho}+\frac{4}{3 S_{1} \operatorname{Re}}\left(\frac{\partial^{2}}{\partial r^{2}}+\frac{1}{r} \frac{\partial}{\partial r}-\frac{1}{r^{2}}\right)\right] \tilde{v} \\
&-\bar{\rho} \bar{u} \tilde{v}_{x}-\frac{\partial \tilde{p}}{\partial r} \\
& \mathrm{i} \alpha \tilde{\rho}=\tilde{\rho}_{x} \\
& \mathrm{i} \alpha\left(-\frac{1}{\alpha} \tilde{\rho} \tilde{u}+\bar{u} \tilde{\rho}\right)=-\left(\bar{\rho}^{\prime}+\frac{\bar{\rho}}{r}+\bar{\rho} \frac{\partial}{\partial r}\right) \tilde{v}+\mathrm{i} \omega \tilde{\rho} \\
&+\left(i \omega \bar{\rho} f+\frac{1}{S_{1} \operatorname{RePr} \operatorname{RePr}}\left[f \frac{\partial^{2}}{\partial r^{2}}+\left(2 f^{\prime}+\frac{f}{r}\right) \frac{\partial}{\partial r}\right.\right. \\
&\left.\left.+\left(f^{\prime \prime}+\frac{f^{\prime}}{r}\right)\right]\right) \tilde{\rho} .
\end{aligned}
$$

Here, the temperature perturbation $\tilde{T}$ has been eliminated by applying the algebraic linearized equation of state. Furthermore, in order to write the equations resulting from this elimination more succinctly, a new variable, $f$, is defined in terms of mean quantities such that

$$
T=-\frac{\left(S_{1}-1\right) \bar{T}+1}{\left(S_{1}-1\right) \bar{\rho}} \rho=-\frac{1}{\left(S_{1}-1\right) \bar{\rho}^{2}} \rho=f(r) \rho .
$$

\subsubsection{Matrix notation}

Equations (4.2) were written in a special form so that the eigenvalue $\alpha$ appears only on the left-hand side. This was to enable us to represent the eigenvalue problem (4.2) in an efficient matrix notation as follows.

$$
\mathrm{i} \alpha \boldsymbol{M q}=\boldsymbol{L q} .
$$

In this equation, $\boldsymbol{q}$ denotes the state vector of our seven independent variables

$$
\boldsymbol{q}=\left[\begin{array}{lllllll}
\tilde{u} & \tilde{u}_{x} & \tilde{v} & \tilde{v}_{x} & \tilde{\rho} & \tilde{\rho}_{x} & \tilde{p}
\end{array}\right]^{T} .
$$


In (4.4), $\boldsymbol{M}$ is the coefficient matrix of the state variables appearing on the left-hand side of (4.2).

$$
\boldsymbol{M}=\left[\begin{array}{ccccccc}
1 & 0 & 0 & 0 & 0 & 0 & 0 \\
0 & -\frac{4}{3 S_{1} R e} & 0 & 0 & 0 & 0 & 1 \\
0 & 0 & 1 & 0 & 0 & 0 & 0 \\
0 & 0 & 0 & -\frac{1}{S_{1} R e} & 0 & 0 & 0 \\
0 & 0 & 0 & 0 & 1 & 0 & 0 \\
\bar{\rho} & 0 & 0 & 0 & \bar{u} & 0 & 0 \\
0 & 0 & 0 & 0 & 0 & \frac{f}{S_{1} \operatorname{RePr}} & 0
\end{array}\right] .
$$

Similarly, $\boldsymbol{L}$ is the coefficient matrix of the state variables appearing on the right-hand side of (4.2). This matrix takes the form

$$
\boldsymbol{L}=\left[\begin{array}{ccccccc}
0 & 1 & 0 & 0 & 0 & 0 & 0 \\
L_{21} & L_{22} & L_{23} & L_{24} & L_{25} & 0 & 0 \\
0 & 0 & 0 & 1 & 0 & 0 & 0 \\
0 & L_{42} & L_{43} & L_{44} & 0 & 0 & L_{47} \\
0 & 0 & 0 & 0 & 0 & 1 & 0 \\
0 & 0 & L_{63} & 0 & L_{65} & 0 & 0 \\
0 & 0 & L_{73} & 0 & L_{75} & L_{76} & 0
\end{array}\right]
$$

where the non-zero elements are as follows:

$$
\begin{aligned}
& L_{21}=\mathrm{i} \omega \bar{\rho}+\frac{1}{S_{1} R e}\left(\frac{\partial^{2}}{\partial r^{2}}+\frac{1}{r} \frac{\partial}{\partial r}\right), \quad L_{22}=\bar{\rho} \bar{u}, \quad L_{23}=-\bar{\rho} \bar{u}^{\prime}, \\
& L_{24}=\frac{1}{3 S_{1} R e}\left(\frac{\partial}{\partial r}+\frac{1}{r}\right), \quad L_{25}=-\frac{1}{F r^{2}}, \\
& L_{42}=\frac{1}{3 S_{1} \operatorname{Re}} \frac{\partial}{\partial r}, \quad L_{43}=\mathrm{i} \omega \bar{\rho}+\frac{4}{3 S_{1} \operatorname{Re}}\left(\frac{\partial^{2}}{\partial r^{2}}+\frac{1}{r} \frac{\partial}{\partial r}-\frac{1}{r^{2}}\right), \\
& L_{44}=-\bar{\rho} \bar{u}, \quad L_{47}=-\frac{\partial}{\partial r}, \\
& L_{63}=-\bar{\rho}^{\prime}-\bar{\rho}\left(\frac{\partial}{\partial r}+\frac{1}{r}\right), \quad L_{65}=\mathrm{i} \omega \tilde{\rho}, \\
& L_{73}=-\bar{\rho} \bar{T}^{\prime}, \\
& L_{75}=\mathrm{i} \omega \bar{\rho} f+\frac{1}{S_{1} \operatorname{RePr} r}\left[f \frac{\partial^{2}}{\partial r^{2}}+\left(2 f^{\prime}+\frac{f}{r}\right) \frac{\partial}{\partial r}+\left(f^{\prime \prime}+\frac{f^{\prime}}{r}\right)\right], \\
& L_{76}=-\bar{\rho} \bar{u} f .
\end{aligned}
$$

Standard eigenvalue routines (such as the QR-algorithm) can then be used on the discretized versions of the matrix operators $\boldsymbol{L}$ and $\boldsymbol{M}$. 


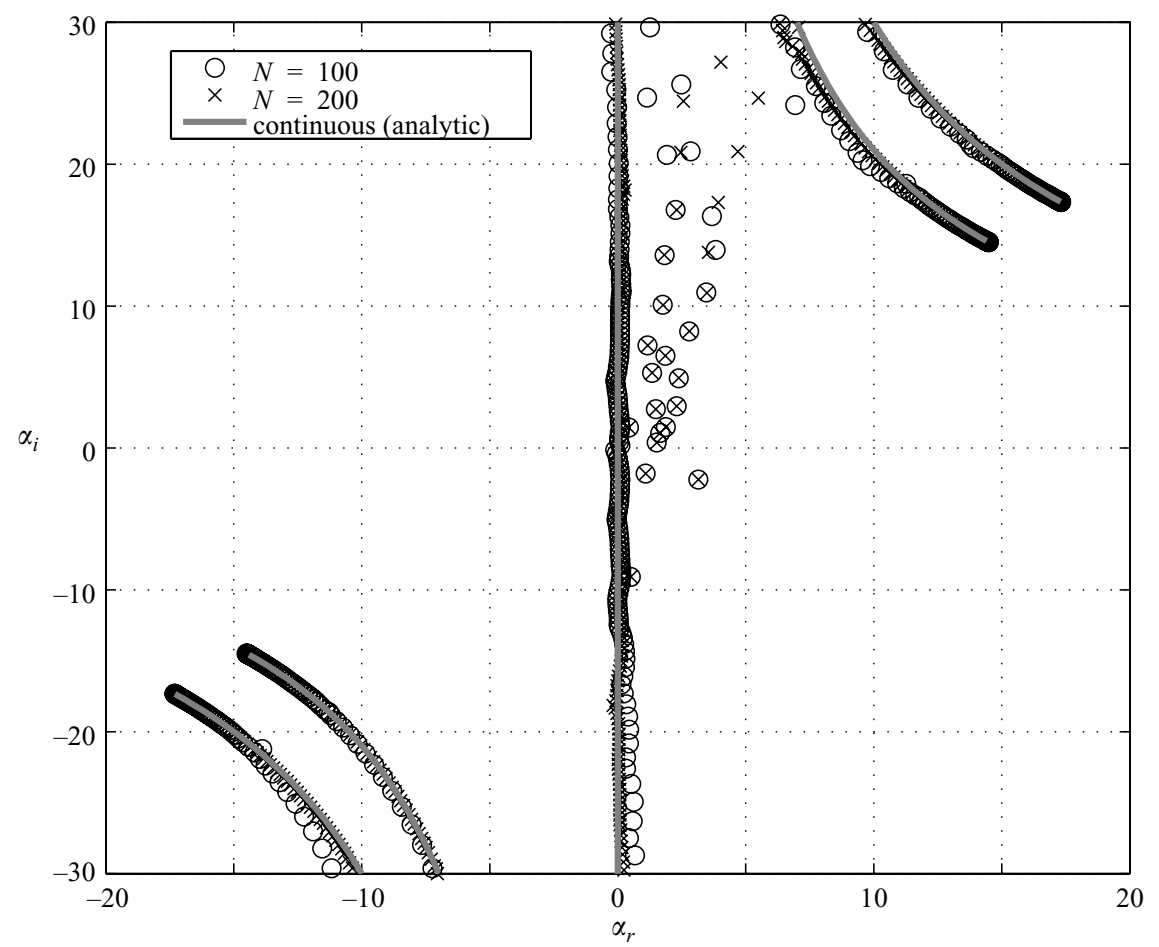

FIGURE 11. Spectrum of the complex eigenvalues $\alpha$ of (4.4). This spectrum was calculated using the parameters $\operatorname{Re}=100, S_{1}=4.0, F r=\infty, \operatorname{Pr}=0.7$ and $\omega=1.5$.

\subsection{Eigenvalue spectrum}

Because the extent of the radial coordinate direction is semi-infinite, we expect a discrete and a continuous spectrum when solving for the eigenvalues of the linear stability operator. Discrete modes are confined to the part of the radial coordinate direction that shows variations in the mean velocity, density and temperature profiles. The continuous modes, on the other hand, have non-zero contributions in the free stream.

Figure 11 shows the complete spectrum of eigenvalues of the numerical approximation to (4.4) plotted in the complex $\alpha$-plane. The symbols on the plot are obtained by discretizing the linear operator in the radial coordinate using a spectral method. The curves shown in grey, however, are an analytic representation of the continuous portion of the spectrum, a derivation of which is given in the Appendix. As figure 11 demonstrates, the analytic curves become increasingly better approximated as the resolution of the spectral discretization is increased.

In addition to the continuous modes, figure 11 shows a spectrum of discrete modes in the region $0<\alpha_{r}<5$ which do not appear to be associated with the grey curves. The eigenvalues associated with the least-damped discrete modes lie in the region $\alpha_{i}<10$, and in each of these cases, we observe that the spectral resolution is sufficient to determine the location of the eigenvalue precisely.

A Reynolds number of $R e=100$ was selected to generate figure 11 so as to clearly display both the discrete and continuous portions of the spectrum on the same plot. For the transitioning jets in the previous section, the Reynolds numbers were 

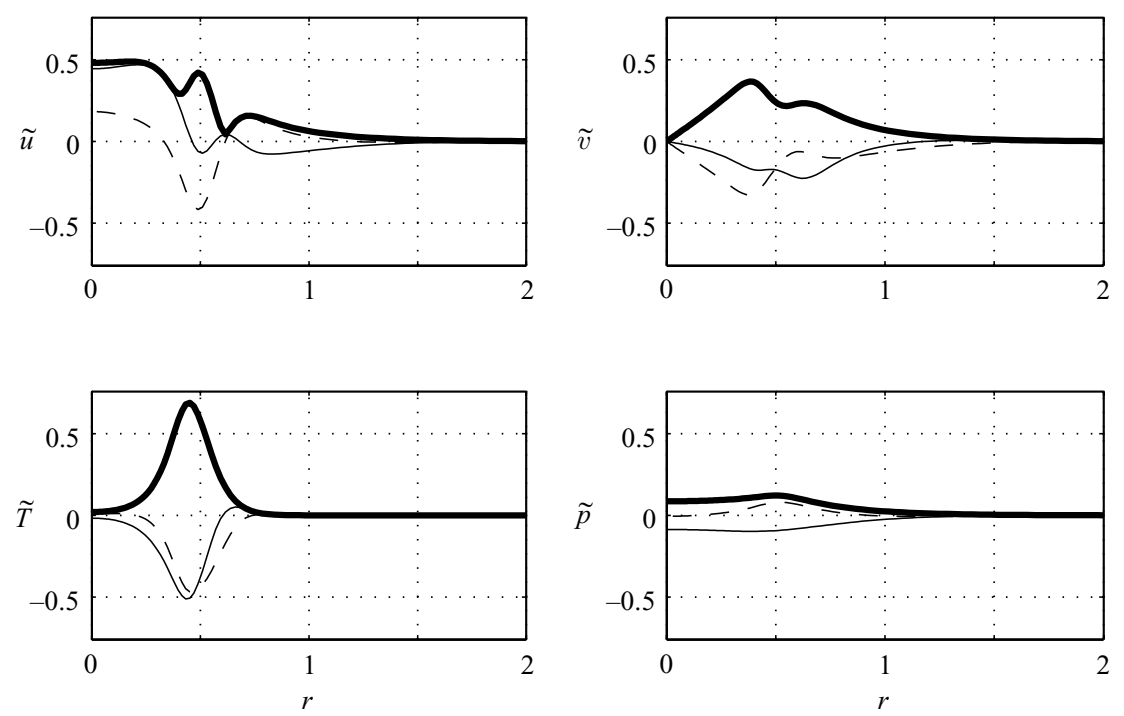

FIGURE 12. Eigenfunction associated with the unstable eigenvalue of the discrete spectrum for which $\alpha_{i}<0$ and $\partial \omega / \partial \alpha>0$. The solid, dashed and heavy black lines indicate the real part, the imaginary part and magnitude of the eigenfunction, respectively. $\alpha=(3.153178,-2.227813 i)$.

$R e=1000$, in which case the portions of the continuous spectrum having hyperbolic form (see the Appendix) move further away from the origin.

The discrete spectrum shown in figure 11 appears to contain two eigenvalues for which $\alpha_{i}<0$. In the case of negative $\alpha_{i}$, the associated eigenfunction amplifies in the downstream direction according to (4.1). It is premature, however, to conclude that these eigenvalues are unstable, because the group velocity $\partial \omega / \partial \alpha$ must also be considered. As will be detailed in a following section, the group velocity for one of these modes is negative while the other is positive. A negative group velocity implies upstream energy propagation, and for the currently considered case of $\alpha_{i}<0$ this upstream energy propagation is damped. Therefore, there is only one discrete mode for which $\alpha_{i}<0$ and $\partial \omega / \partial \alpha>0$ which may be called unstable. Figure 12 shows the components $\tilde{u}, \tilde{v}, \tilde{T}$ and $\tilde{p}$ of the eigenfunction associated with the unstable eigenvalue.

\subsection{Absolute stability analysis}

When analysing the spatial stability of shear flows it is prudent to investigate the potential for absolutely unstable modes. An absolute instability exists if a deltafunction forcing in space and time results in an upstream and downstream propagating disturbance that eventually contaminates the entire flow domain. Mathematically, absolute instabilities manifest themselves by the occurrence of pinch points between spatial spectral branches in the unstable half of the complex $\alpha$-plane. Brigg's method is the standard technique for analysing shear flows for the existence of absolutely unstable behaviour.

\subsubsection{Brigg's method}

Starting with a linear partial differential equation forced by delta functions in space and time

$$
D\left(-\mathrm{i} \frac{\partial}{\partial x}, \mathrm{i} \frac{\partial}{\partial t}\right) G(x, t)=\delta(x) \delta(t)
$$




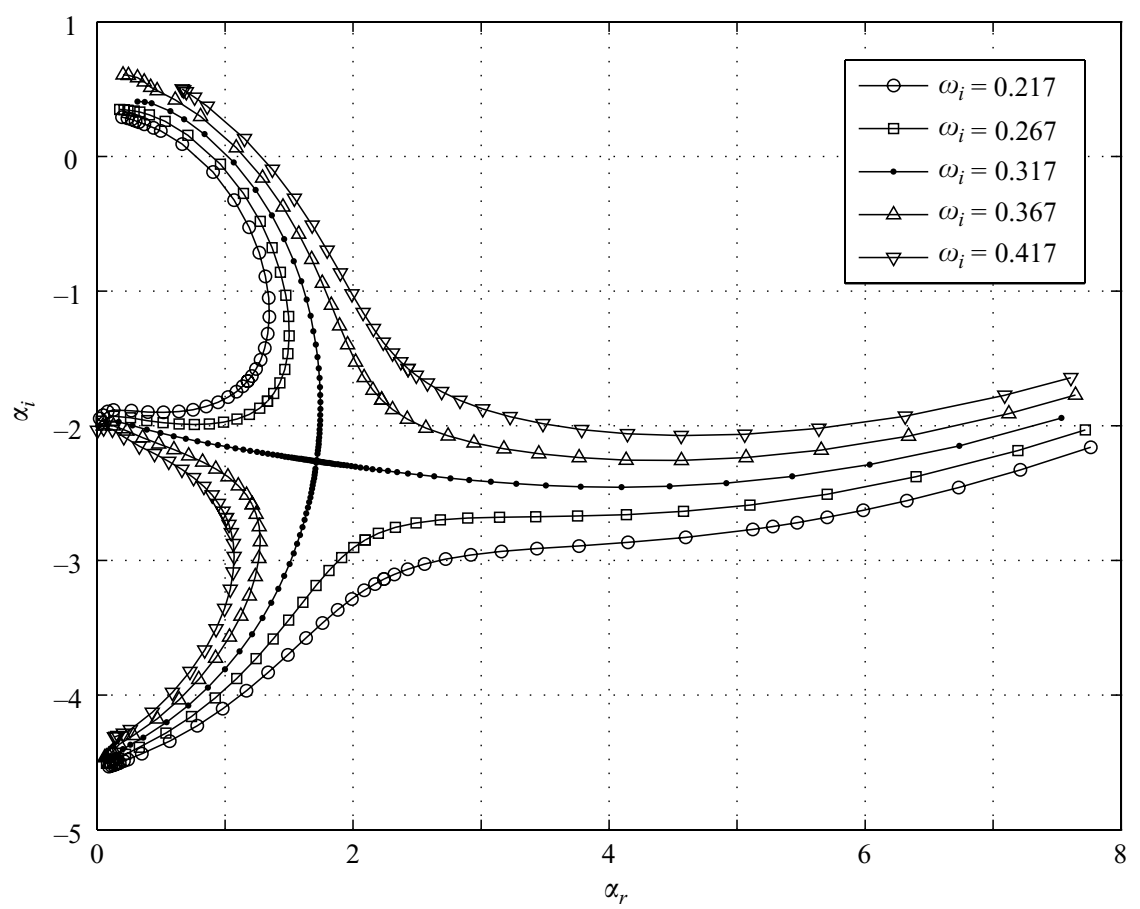

FIGURE 13. Pinch point visualized by mapping lines parallel to the real axis in the complex $\omega$-plane into the complex $\alpha$-plane.

we are interested in the long-time $(t \rightarrow \infty)$ behaviour at $x=0$. Using a joint FourierLaplace transform of the equation we can formulate the solution as

$$
G(x, t)=\frac{1}{4 \pi^{2}} \int_{L} \int_{F} \frac{\exp (\mathrm{i} \alpha x-\mathrm{i} \omega t)}{D(\alpha, \omega)} \mathrm{d} \alpha \mathrm{d} \omega,
$$

with $D(\alpha, \omega)$ as the dispersion relation associated with the homogeneous part of (4.9). In order to evaluate the integral asymptotically we start with a temporal integration contour $L:-\infty<\omega_{r}<\infty$ and $\omega_{i}$ sufficiently high. We subsequently lower the contour by decreasing $\omega_{i}$ until the spatial integration contour $F$ is pinched between two branches of the spatial stability problem. It is important to keep in mind that the two spatial branches have to correspond to an upstream and downstream propagating mode. The value of the temporal growth rate $\omega_{i}^{0}$ at which the temporal integration contour cannot be lowered any farther determines the absolute growth rate of the flow. If $\omega_{i}^{0}$ is positive, the flow is absolutely unstable; if $\omega_{i}^{0}$ is negative, the flow is absolutely stable, but may be convectively unstable. Pinch points in the complex $\alpha$-plane map to corresponding branch points in the $\omega$-plane through the generalized dispersion relation. For more details on Brigg's method and the computation of absolute instabilities, see Briggs (1964).

Up to this point, we have used $R e=100$ in order to illustrate the spatial eigenvalue spectrum. We now consider the parameter set corresponding to the simulations, for which $R e=1000$. The mean velocity and density profiles are taken from the inlet boundary conditions displayed in figure 2 , where $R / \theta_{u}=12.5, R / \theta_{\rho}=12.5$ and $S_{1}=4$.

The mapping of the $\omega$-plane under the dispersion relation $D(\alpha, \omega)$ around the resulting pinch point is visualized in figure 13. In the $\alpha$-plane, the location of the pinch point is $\alpha^{0}=1.715-2.264 i$. The contours in figure 13 were drawn by mapping 
lines parallel to the real axis in the $\omega$-plane into the $\alpha$-plane. For example, since the branch point was found to be at $\omega^{0}=1.493+0.317 i$ in the $\omega$-plane, the lines marked with filled dots in figure 13 represent the map of the horizontal line $\omega_{i}=0.317$ in the $\omega$-plane onto the $\alpha$-plane. Likewise, the contours marked with upward pointing triangles in figure 13 are the map of the horizontal $\omega$-plane line $\omega_{i}=\omega_{i}^{0}+0.05=0.367$.

Since the imaginary part $\omega_{i}^{0}=0.317$ is positive, the flow is predicted to be absolutely unstable, which we have observed to be true by direct numerical simulation. The corresponding Strouhal number can be calculated from $\omega_{r}^{0}$ as follows:

$$
S t=\frac{\omega_{r}^{0} d}{2 \pi u_{j}}=\frac{1.493 \times 1.0}{2 \pi \times 1.0} \approx 0.238 .
$$

This represents a relative error of $-4.8 \%$ compared to the measured dominant Strouhal number of the previous section (see table 2, simulation A 2). A possible explanation for this discrepancy is that the axially evolving mean profiles have a large effect on the dominant frequency predicted.

\subsection{Axial development of stability properties}

In this section, we study how the local stability properties of the jet change as the jet develops downstream. We approach this problem from two directions. First, we study how the stability properties change as the parameters describing the model top-hat mean profiles are systematically varied. Secondly, we replace the model mean profiles with mean profiles measured directly from the simulations.

It is important to note that both of these analyses employ the parallel-flow assumption and so provide information only about local stability properties about a given axial location. In this way, the spreading of the jet is taken into account indirectly, inasmuch as it modifies the mean profiles about which we linearize. This approach is similar to that used by Raynal et al. (1996) for the variable-density plane jet. It is also possible to consider the effect of jet spreading directly through the addition of a slowly varying scale, and in this way obtain global instability modes (see Lesshafft et al. 2006). It is thought, however, that the onset of local absolute instability is a necessary condition for the onset of global absolute instability (see Chomaz 2005).

\subsubsection{Parametric study}

The parameters $R_{u} / \theta_{u}, R_{\rho} / \theta_{\rho}, R e, S_{1}$ and $1 / F^{2}$ form a five-dimensional parameter space. In order to study how the complex frequency $\omega^{0}$ and complex wavenumber $\alpha^{0}$ of the absolute instability change as these parameters vary, the pinch point analysis of $\S 4.3 .1$ could be repeated for every point in the five-dimensional parameter space. Because this is computationally impractical, we restrict the domain of our parametric study to two-dimensional 'slices' through the larger five-dimensional space. This is done by varying pairs of the five parameters while the other three are held constant. Figure 14 illustrates this graphically for the case of a three-dimensional parameter space. The cube represents the entire parameter space, and the dashed lines indicate the planes along which we measure. Note that only two of the three possible planes are shown (the other being the $S_{1}-1 / F r^{2}$ plane). For the five-dimensional case, there are ten such slices, all of which are mutually perpendicular to each other.

Along each of the two-dimensional planes, the branch point $\omega^{0}$ corresponding to the pinch point $\alpha^{0}$ is measured, as previously mentioned. Figure 15 displays contours of the non-dimensional frequency $S t$ which is calculated from $\omega_{r}^{0}$. Each subplot in the figure corresponds to one of the ten possible planes discussed above. The five 


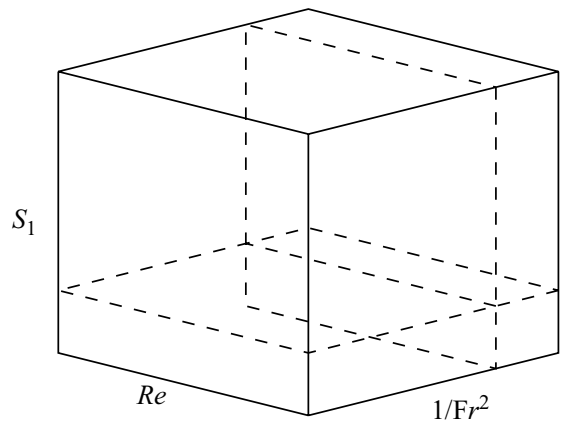

FIGURE 14. Pairwise variation of flow parameters.
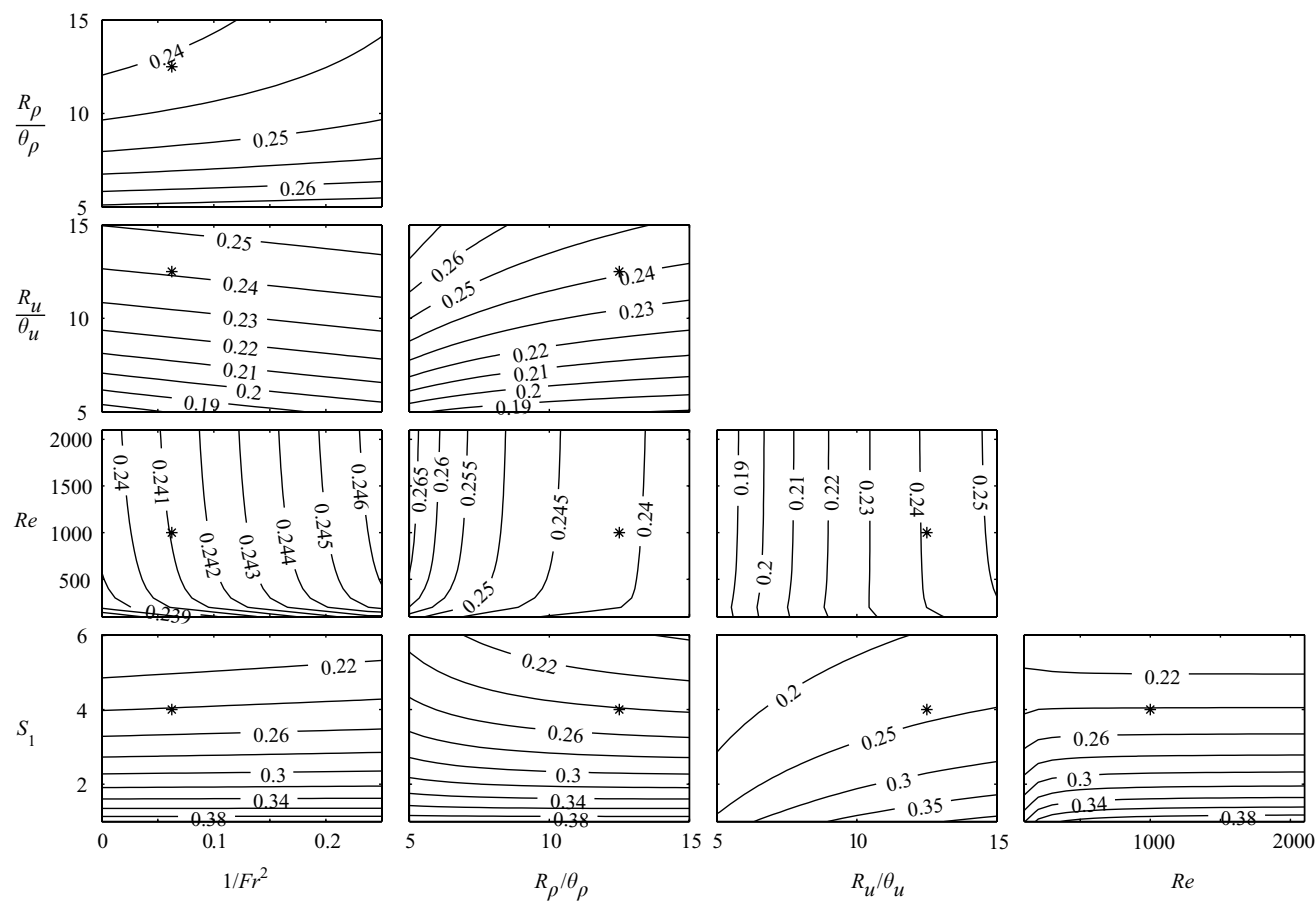

FIGURE 15. Contours of $S t$ for primary instability. The asterisk denotes the intersection point of all the planes.

parameters are arranged along the horizontal and vertical axes in such a way that the resulting triangular matrix encompasses all of the possible pairwise combinations.

The contour lines in figure 15 provide a convenient way of establishing the relative importance of each pair of parameters: if the contour lines in a subplot are mostly horizontal (as is the case in the subplot $S_{1}-1 / F r^{2}$ ), the jet frequency is more sensitive to the parameter along the vertical axis. The converse is also true: if the contours are mostly vertical, the parameter along the horizontal axis is more important in determining the jet frequency. In this way, the contour lines literally 'point' to the more important parameter.

The rationale for pairwise variation of parameters is to use information obtained about the relative importance of each pair of parameters to develop an overall ranking 


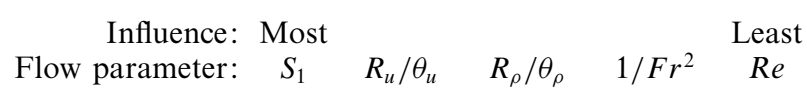

TABLE 3. Ranking of parameters according to their influence upon $\omega^{0}$ for jets of sufficiently high Reynolds number $(\operatorname{Re}>500)$.
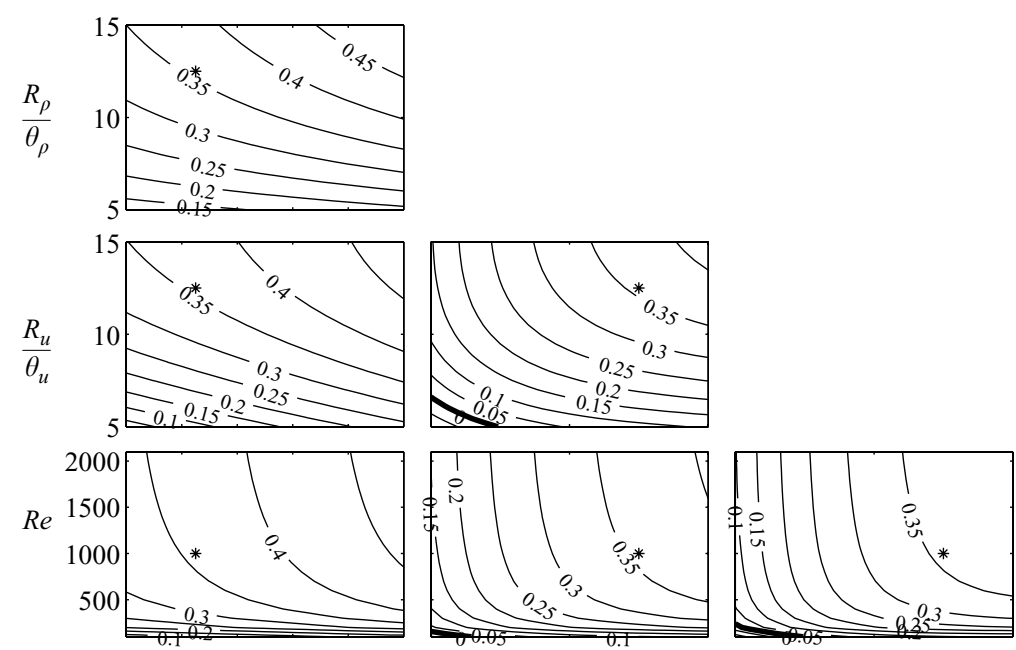

$S_{1}$
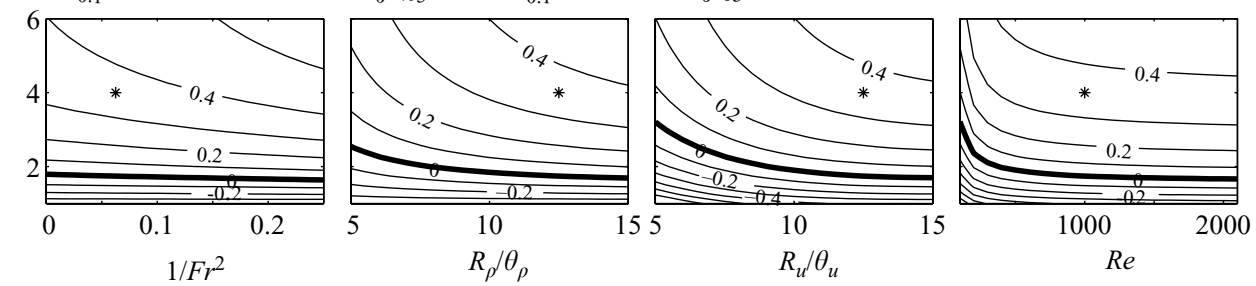

FIGURE 16. Contours of temporal growth rate $\left(\omega_{i}^{0}\right)$ for primary instability. The asterisk denotes the intersection point of all the planes.

of the five parameters. Because the bottom row of figure 15 contains mostly horizontal contour lines, we conclude that $S_{1}$ is the most important parameter in determining the frequency of the absolute instability. Note also that the jet frequency is not sensitive to $R e$ as long as $R e$ exceeds a threshold of about 500. Above this threshold, the parameters can be ranked as shown in table 3. Below the threshold of $R e=500$, however, the jet frequency becomes sensitive to the Reynolds number, so that in this case, $R e$ should be ranked between $R_{u} / \theta_{u}$ and $R_{\rho} / \theta_{\rho}$. In either case, we note that the jet frequency is relatively insensitive to buoyancy in the range $2.0<F r<\infty$.

Figure 16 displays contours of the temporal growth rate $\omega_{i}^{0}$ using the same technique. For high Reynolds numbers, we again find the parameter ranking to be the same as shown in table 3 , with the possible exception that $R_{u} / \theta_{u}$ and $R_{\rho} / \theta_{\rho}$ have relatively equal effects on the temporal growth rate of the absolute instability. Low Reynolds numbers, however, appear to have a strong effect, so that for $R e<500, R e$ becomes the most important parameter determining the temporal growth rate.

While viscous effects become important below a threshold of $R e=500$, the ranking given in table 3 remains a consistent guide for jets with $R e>500$, and this provides some rationale for the selection of $R e=1000$ in the simulations of the first part 

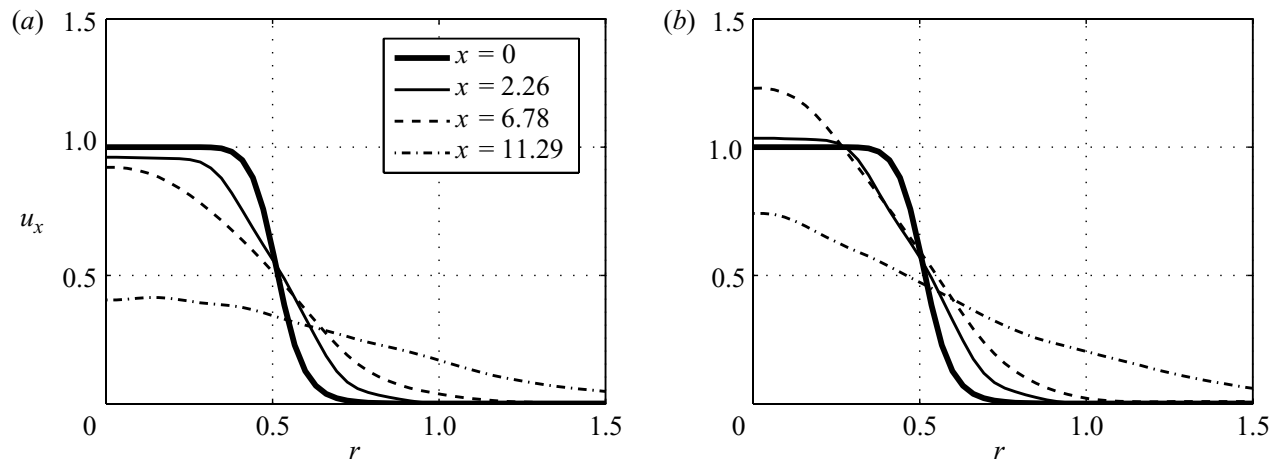

FIGURE 17. Mean axial velocity profiles measured from $(a)$ the zero gravity jet and $(b)$ the buoyant jet.

of this paper. To summarize this case, we have found that the characteristics of the absolute instability depend strongly on the density ratio $S_{1}$, show a somewhat lesser dependence on shear- and density-layer thicknesses, have a surprisingly weak dependence on buoyancy, and have almost no dependence on Reynolds number for jets with $R e>500$.

Finally, note that the flow parameters of this section have been written so that the spreading and relaxation of a jet as it develops downstream will result in an eventual decrease in all of these quantities. This means that a jet with inlet conditions given by the asterisks in figure 16 will migrate down and to the left in each subplot. The heavy black lines in this figure indicate the contour of zero temporal growth rate and represent the boundary between absolute and convective instability. We therefore expect the jet to transition from absolute instability to convective instability as the boundary displayed in figure 16 is crossed at some downstream location within the jet. Note that because this boundary separates two regimes of different types of instability, it is not the same as a neutral curve which, by definition, separates a regime of instability from a regime of stability.

\subsection{Mean profiles from DNS}

Figure 17 shows the local mean axial velocity profiles at various downstream locations for the non-buoyant and buoyant jets. For the zero gravity jet, the jet centreline velocity decreases monotonically with respect to $x$. Also, as the jet develops downstream, the inlet top-hat profile gradually relaxes to a fully developed jet profile, as expected. For the buoyant jet, the centreline velocity increases for a distance downstream before starting to decay.

Figure 18 displays the corresponding mean density profiles. In every case, the density profile tends to spread radially more than the corresponding axial velocity profile. Also, instead of relaxing directly into a fully developed profile, the mean density profile first develops a 'plateau' near the centre of the transition layer, the origin of which will be discussed in the following section.

Performing a pinch-point analysis using the mean profiles measured at each axial location $x$, we obtain the development of the frequency and temporal growth rate of the absolute instability as a function of axial distance $x$ (figure 19). As is evident from figure 19, the buoyancy can play a role in maintaining the frequency of the absolute instability over the length of the jet. Recall from the parametric study in the previous section that the buoyancy has little direct effect on the frequency of 
(a)

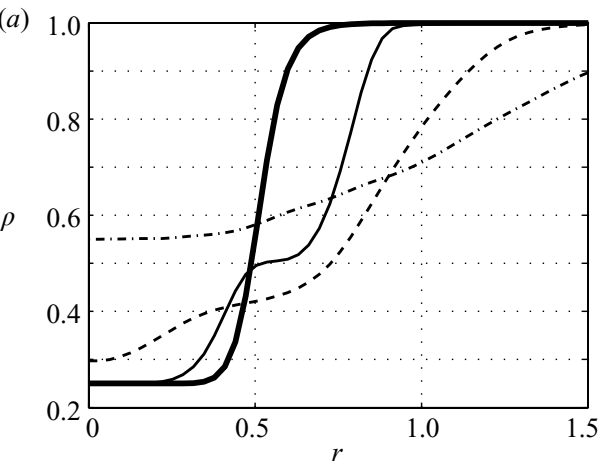

(b)

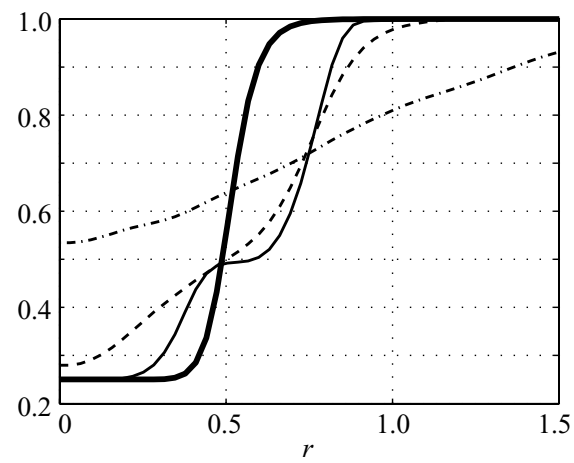

FIGURE 18. Mean density profiles measured from $(a)$ the zero gravity jet and $(b)$ the buoyant jet.

(a)

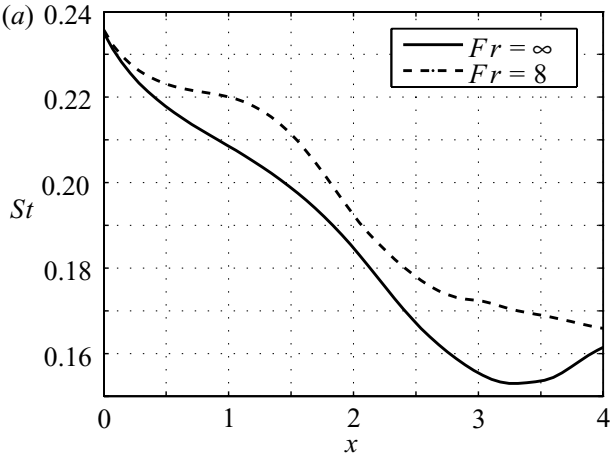

(b)

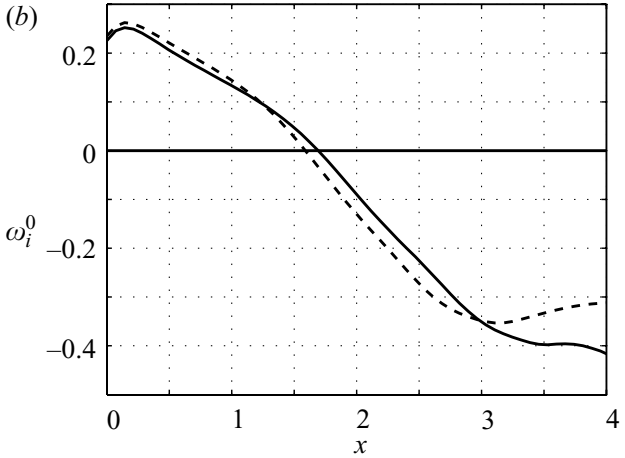

FIGURE 19. (a) Frequency and $(b)$ temporal growth rate of the local absolute instability as a function of $x$, derived from the mean profiles measure from DNS. A pocket of local absolute instability is observed from the jet inlet to approximately 1.5 diameters downstream.

the absolute instability. The effect observed in figure 19 must then be caused by an indirect effect of buoyancy. For instance, the acceleration due to buoyancy increases the local Reynolds number, as well as decreases the thickness of the shear layer since the shape of the axial velocity profile changes.

As is evident from this figure, buoyancy does not have much of an effect on the growth rate. This figure is interesting, however, because it shows how the jet transitions from absolute to convective instability. The region above the heavy black line represents the regime of absolute instability whereas the region underneath it represents the regime of convective instability. From this plot, we see clear evidence of a pocket of absolute instability from the jet inlet to around $x=1.5$. Since the jet flows from the pocket of absolute instability into the region of convective instability, the jet can be thought of from a systems standpoint as a self-sustaining oscillator which feeds into an amplifier (see Huerre 2000).

\subsection{Effects of mixing}

To further explain the invariance of the length of the pocket of absolute instability with respect to buoyancy as observed in figure 19, we consider in this section the influence of the formation of the previously mentioned plateau in the mean density 
(a)

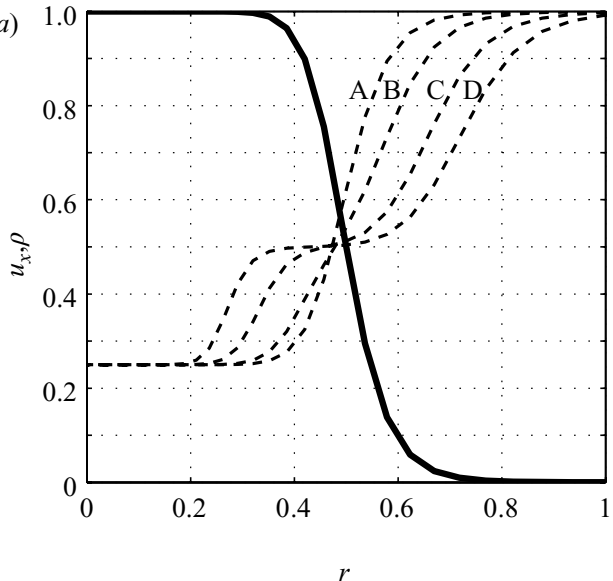

(b)

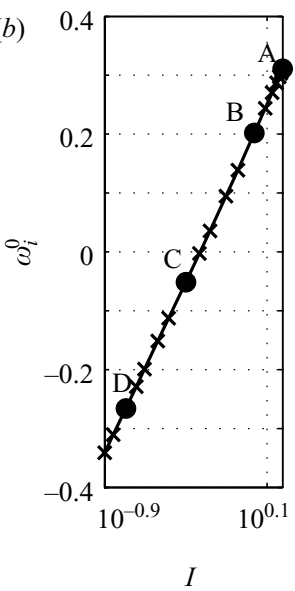

(c)

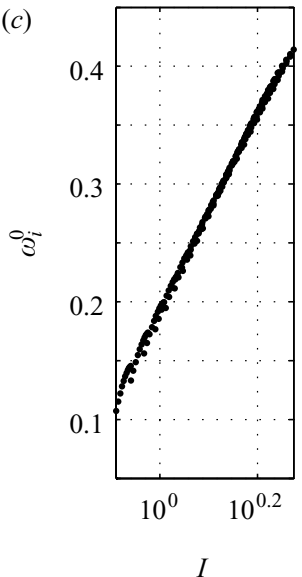

FIGURE 20. (a) axial velocity profile (solid) and family of density profiles (dashed) modelling mixing, $(b)$ temporal growth rate $\omega_{i}^{0} v s$. alignment $I$ for the family of profiles shown in $(a)$, and $(c)$ temporal growth rate $v s$. alignment for variable shear- and density-layer thicknesses.

profile. This plateau arises because of the roll-up of Kelvin-Helmholtz vortices and is sustained by strong mixing occurring in their cores.

In figure 20( $a$ ) we consider a family of density profiles formed by the superposition of two radially offset tanh profiles. In this way, the gradient of the density profile is lessened at the point where the axial velocity gradient is strongest. To quantify the total alignment of the profiles, we define an integral quantity, $I$, such that:

$$
I=-\int_{0}^{\infty} \frac{\partial u_{x}}{\partial r} \frac{\partial \rho}{\partial r} r \mathrm{~d} r .
$$

Figure 20(b) shows that the temporal growth rate $\omega_{i}^{0}$ is proportional to the logarithm of this alignment term $I$. Since $\omega$ appears in the exponent of (4.1) this means that the rate of growth of the total amplitude of the absolute instability is proportional to $I$.

The dots labelled A, B, C and D in figure 20(b) correspond to the density profiles labelled similarly in figure $20(a)$. Note that the formation of even a rather modest plateau as in profile $\mathrm{C}$ is enough to cause the jet to switch from absolute to convective instability, as $\omega_{i}^{0}$ becomes negative for this case. Since profile $\mathrm{C}$ is representative of those measured from DNS, we suggest that the formation of the plateau could be responsible for the development of $\omega_{i}^{0}$ within the pocket of absolute instability, masking other weaker effects such as those due to buoyancy.

To further test the idea that the growth rate of the amplitude of the absolute instability is proportional to the alignment term $I$, we consider jets with varying shear- and density-layer thicknesses, but with no density plateau. In the ranges $10<R_{u} / \theta_{u}<15$, and $5<R_{\rho} / \theta_{\rho}<15$, corresponding to the top half of the $R_{u} / \theta_{u}-R_{\rho} / \theta_{\rho}$ subplot in figure 16, the data for $\omega_{i}^{0}$ again collapse to a straight line, as shown in figure $20(c)$.

A possible physical interpretation of the alignment term $I$ involves baroclinic torque. If we assume that the strength of any initial axial pressure perturbations, as measured by $\partial p / \partial x$, are proportional to $\partial u_{x} / \partial r$, then $I$ will be proportional to the baroclinic torque generated by the interaction of these pressure perturbations with the mean density gradient. Therefore, in addition to characterizing the alignment of 
the mean profiles, $I$ might also be interpreted as the total 'availability' of baroclinic torque within the domain.

\section{Conclusions and recommendations}

We have verified, both through DNS and linear stability analysis, that variabledensity jets, both with and without the presence of a buoyant force, exhibit absolute instability. The absolutely unstable jet has a natural frequency at which it generates a continuous series of vortex rings. The linear theory predicted this natural frequency to within $5 \%$ of that measured from DNS. Furthermore, using mean profiles taken from DNS data, this local absolute instability was shown to be confined to a pocket near to the nozzle. Therefore, variable-density jet dynamics can be thought of as an upstream oscillator driving a downstream amplifier.

Furthermore, the parametric study showed that in variable density jets, buoyancy does not have a strong direct effect on the absolute instability. Therefore, we conclude that if buoyancy does play a role in changing the local stability properties of a variable-density jet, it must be an indirect effect. For example, the acceleration due to buoyancy will increase the local Reynolds number. More subtly, this acceleration also changes the shape of the mean profiles, producing thinner shear layers. Consulting figure 15 , both of these changes result in higher-frequency self-sustaining oscillations for the buoyant jet, which was observed in figure 19.

From the DNS results, we observed that buoyancy does have the effect of producing more abrupt transition to turbulence. As discussed below, it is thought that this is due in part to the suppression or alteration of the secondary instability, since the side jets present in the non-buoyant case were absent in the buoyant case. To test this hypothesis, however, further work is required. The more abrupt transition to turbulence in the case of the buoyant jet is in agreement, however, with the observation of Bahadori et al. (1992) that instabilities appear first at the flame tip, whereas microgravity (approximately non-buoyant) flame instabilities incorporate the entire flame.

The experiments of Bahadori et al. (1992) focused on propane/air flames, for which $S_{1}<1.0$, and so absolute instability is not expected. They comment, however, that they repeated the experiments for methane flames and observed qualitatively similar results, i.e. a marked increase in microgravity flame length (see Hegde et al. 1994). This is surprising because stability theory applied to related cases suggests that absolute instability will be present in the case of the methane jet no matter the gravity level. Moreover, the mixing due to the self-sustained oscillations in the methane jet will be nearly the same in the non-buoyant and buoyant cases, since $S_{1}$ is a more dominant parameter in determining the frequency than $F r$. This consideration tends to support the experimental observations of Idicheria et al. (2003) who saw no increase in flame height in microgravity conditions.

Finally, although we conclude that the increase in flame length observed by Bahadori et al. (1992) and Hegde et al. (1994) cannot be due to a suppression of mixing due to the primary instability, this effect may still be due to the presence of a secondary instability. For instance, in the microgravity jet, we observed the formation of side jets which eject fuel from the centre of the jet. This would produce wider flames in the near field, which then could persist further downstream.

In fact, in this paper, we have seen strong evidence of secondary instability, including the appearance of coherent structures in figure 3 and the initial exponential growth of the radial and axial components of vorticity observed in figure 6 . Furthermore, this type of secondary instability has been observed in jet diffusion flames in the laboratory 
(see Demare \& Baillot 2001). To more fully understand these secondary instabilities, further theoretical development is required. In computing the complete spatial spectrum and deriving analytic forms for the continuous portions of this spectrum, however, this paper has constructed a solid theoretical framework on which to build.

This research was supported by NASA Grant NAG3-2517, issued by the NASA Glenn Research Center. Support for the direct numerical simulations was provided by a grant of HPC resources from the Arctic Region Supercomputing Center at the University of Alaska Fairbanks as part of the Department of Defense High Performance Computing Modernization Program. The authors would like to thank John Kramlich and George Kosály for many helpful discussions during the course of this research. We would also like to thank the anonymous reviewers for several helpful suggestions.

\section{Appendix. Continuous spectrum}

Because of the unbounded nature of the domain in the $r$-direction, we expect and confirm the presence of a continuous spectrum. Eigenfunctions associated with eigenvalues on the continuous spectrum display highly oscillatory behaviour as $r$ tends to infinity; in contrast, eigenfunctions associated with the discrete spectrum are confined to oscillate in the interior of the jet. Because the eigenfunctions on the continuous spectrum oscillate as $r \rightarrow \infty$, the boundary condition at infinity must be relaxed to $\tilde{\rho}, \tilde{u}, \tilde{v}, \tilde{p}$ bounded as $r \rightarrow \infty$ to accommodate and accurately capture the continuous part of the spectrum in our computations.

An analytical expression for the location of the continuous spectrum can easily be derived by taking the limit as $r \rightarrow \infty$ of the linear stability operator. In this limit, the mean profiles have the following properties: $\bar{u}, \bar{u}^{\prime}, \bar{T}, \bar{T}^{\prime} \rightarrow 0, \bar{\rho} \rightarrow 1$ and $\bar{\rho}^{\prime} \rightarrow 0$, and the linearized equations reduce to

$$
\begin{gathered}
-\mathrm{i} \omega \tilde{\rho}=-\mathrm{i} \alpha \tilde{u}-\frac{1}{r} \frac{\partial r \tilde{v}}{\partial r}, \\
-\mathrm{i} \omega \tilde{u}=-\mathrm{i} \alpha \tilde{p}-\frac{1}{F r^{2}} \tilde{\rho}+\frac{1}{S_{1} R e}\left(-\frac{4}{3} \alpha^{2} \tilde{u}+\frac{1}{r} \frac{\partial}{\partial r} r \frac{\partial \tilde{u}}{\partial r}+\mathrm{i} \alpha \frac{1}{3 r} \frac{\partial r \tilde{v}}{\partial r}\right), \\
-\mathrm{i} \omega \tilde{v}=-\frac{\partial \tilde{p}}{\partial r}+\frac{1}{S_{1} R e}\left(-\alpha^{2} \tilde{v}+\frac{4}{3} \frac{\partial}{\partial r} \frac{1}{r} \frac{\partial r \tilde{v}}{\partial r}+\mathrm{i} \alpha \frac{1}{3} \frac{\partial \tilde{u}}{\partial r}\right), \\
-\mathrm{i} \omega f \tilde{\rho}=\frac{1}{S_{1} \operatorname{RePr}}\left(-\alpha^{2} f \tilde{\rho}+f \frac{\partial^{2} \tilde{\rho}}{\partial r^{2}}+\frac{f}{r} \frac{\partial \tilde{\rho}}{\partial r}\right) .
\end{gathered}
$$

We observe that (A $1 d$ ) is a homogeneous ordinary differential equation for $\tilde{\rho}$ only. Furthermore, equations (A 1a)-(A 1c) can be solved in terms of the variables $\tilde{v}$ and $\tilde{\rho}$. It is then straightforward to recast the above equations into the following general form:

$$
L_{1} \tilde{v}=L_{2} \tilde{\rho}, \quad L_{3} \tilde{\rho}=0,
$$

where the linear operators $L_{j}$ are given as follows:

$$
\begin{aligned}
& L_{1}=\left(r^{2} D^{2}-3 r D+\left(\mathrm{i} \omega S_{1} R e-\alpha^{2}\right) r^{2}+3\right)\left(r^{2} D^{2}+r D-\alpha^{2} r^{2}-1\right), \\
& L_{2}=\left(r^{2} D^{2}-3 r D+\left(\mathrm{i} \omega S_{1} R e-\alpha^{2}-\frac{\alpha S_{1} R e}{\omega F r^{2}}\right) r^{2}+3\right)\left(-\mathrm{i} \omega r^{2} D\right), \\
& L_{3}=\left(r^{2} D^{2}+r D+\left(\mathrm{i} \omega S_{1} \operatorname{RePr}-\alpha^{2}\right) r^{2}\right) .
\end{aligned}
$$


From (A 2) we can see that the general solution is the superposition of two classes of solutions. One class is associated with the homogeneous solution $\tilde{v}_{h}$ of (A $2 a$ ), which requires that $\tilde{\rho}=0$. The other class is associated with the homogeneous solution $\tilde{\rho}_{h}$ of $(\mathrm{A} 2 b)$. For this class, (A $2 a, b)$ is satisfied by a particular solution $\tilde{v}_{p}$.

$$
\left[\begin{array}{c}
\tilde{v} \\
\tilde{\rho}
\end{array}\right]=C_{1}\left[\begin{array}{c}
\tilde{v}_{h} \\
0
\end{array}\right]+C_{2}\left[\begin{array}{c}
\tilde{v}_{p} \\
\tilde{\rho}_{h}
\end{array}\right] .
$$

The linear operators given in (A 3) can be factored (this factorization is noncommutative, however) so that it is apparent that solutions take the form of Bessel functions. For instance, the homogeneous solution to $L_{1} \tilde{v}_{h}=0$ is:

$$
\tilde{v}_{h}=C_{1} K_{1}\left(a_{1,2} r\right)+C_{2} r^{2} K_{1}\left(a_{3,4} r\right),
$$

where $K_{1}$ denotes the first-order modified Bessel function of the second kind,

$$
\begin{aligned}
& a_{1,2}= \pm\left(\alpha^{2}\right)^{1 / 2} \\
& a_{3,4}= \pm\left(\alpha^{2}-i \omega S_{1} R e\right)^{1 / 2} .
\end{aligned}
$$

The singularity of $K_{1}$ at $r=0$ is not significant to the present analysis because we are interested only in the limiting behaviour as $r \rightarrow \infty$. The square roots appearing in (A 6) and (A 7), give rise to two pairs of branch cuts, which define the locus of the continuous spectrum. We first consider the branch cuts associated with $a_{3,4}$. Following Schmid \& Henningson (2001), if we define an auxiliary function,

$$
Z=\alpha^{2}-\mathrm{i} \omega S_{1} R e
$$

then

$$
a_{3,4}= \pm Z^{1 / 2} \text {. }
$$

We select the negative real axis of the $Z$-plane for the branch cut of the square root function, which implies that the two branches of $Z^{1 / 2}$ are the left and right-half planes, denoted without loss of generality as $a_{3}$ and $a_{4}$, respectively. In the limit of $r \rightarrow \infty$,

$$
K_{1}\left(a_{3,4} r\right) \sim \sqrt{\frac{\pi}{2 a_{3,4} r}} \exp \left(-a_{3,4} r\right),
$$

which implies that, with the above choice of branch cut, the solution with the coefficient $a_{4}$ is the only one which will satisfy the condition that the solution remain bounded as $r \rightarrow \infty$ (see Ashpis \& Reshotko 1990).

In the $\alpha$-plane, the locus of the continuous spectrum is defined as the map of the selected branch cut in the $Z$-plane, through the auxiliary function (A 8). The real and imaginary parts of (A 8) are:

$$
\alpha_{r}^{2}-\alpha_{i}^{2}<0, \quad 2 \alpha_{r} \alpha_{i}=\omega S_{1} R e
$$

If $\omega$ is positive, (A 11) describes sections of hyperbolas in the first and third quadrants of the $\alpha$-plane, ending at the branch points:

$$
\left(\alpha_{r}, \alpha_{i}\right)=\left( \pm \sqrt{\frac{\omega S_{1} R e}{2}}, \pm \sqrt{\frac{\omega S_{1} R e}{2}}\right)
$$

This corresponds to the outer hyperbola branch cuts shown in grey in figure 11. The branch cuts found along the imaginary axis are derived from (A 6) for which the 

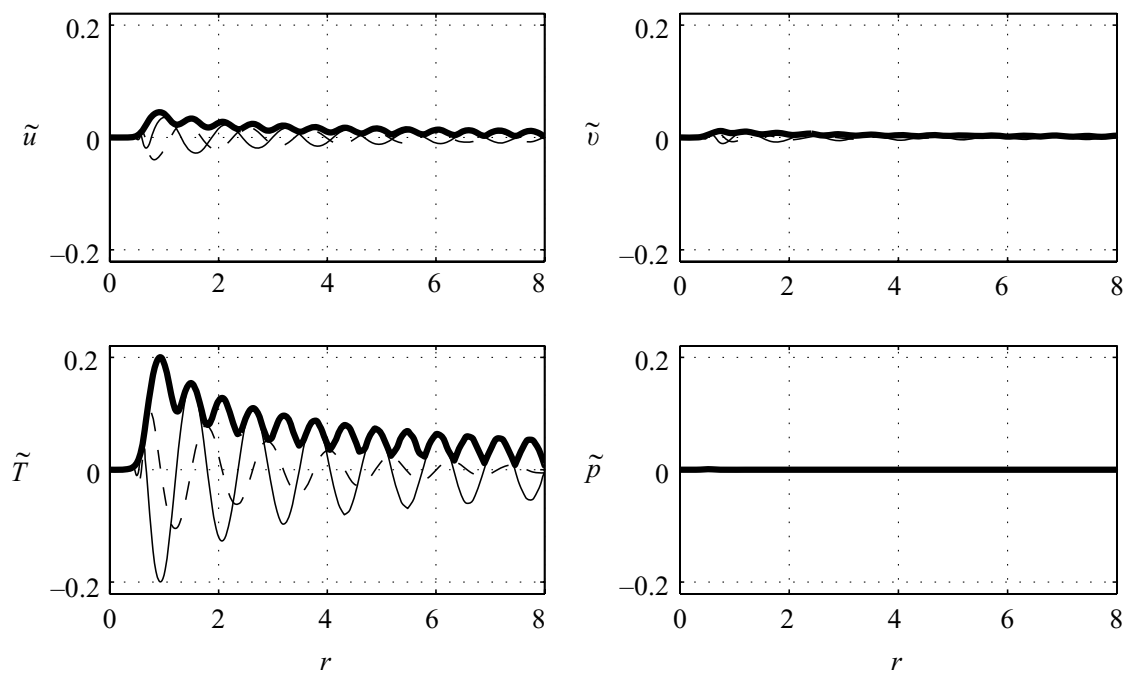

Figure 21. An example of an eigenmode of the continuous spectrum taken from the outer hyperbola branch cuts in figure 11. $\alpha=(16.805786,17.833110 \mathrm{i})$.

auxiliary function is

$$
Z=(\alpha+\epsilon)(\alpha-\epsilon)
$$

so that

$$
a_{1,2}= \pm \lim _{\epsilon \rightarrow 0} Z^{1 / 2}
$$

In this case, the two branch points are infinitesimally close to the origin, but the origin itself remains a regular point which is an important consideration when performing an inverse Fourier transform by contour integration.

The final two portions of the continuous spectrum come from the class of solutions associated with $\tilde{\rho}_{h}$. In this case, the auxiliary function is similar to (A 8), but contains the Prandtl number $\mathrm{Pr}$ as follows:

$$
Z=\alpha^{2}-\mathrm{i} \omega S_{1} \operatorname{RePr} .
$$

In a similar fashion as before, this produces the two hyperbolic branch cuts which are displayed in figure 11 just inside the hyperbolic branch cuts found previously. Note that in the case of $\operatorname{Pr}=1.0$, the two pairs of hyperbolic branch cuts coincide.

Figure 21 displays an example eigenmode of the continuous spectrum in the class $\tilde{v}=\tilde{v}_{h}, \tilde{\rho}=0$ where $\tilde{v}_{h}=r^{2} K_{1}\left(a_{4} r\right)$ as $r \rightarrow \infty$, which corresponds to the outer hyperbola branch cut. As expected, $\tilde{T}=f \tilde{\rho}$ is nearly zero at all radial locations for this case (where $f$ is negative and non-zero for all $r$, see (4.3)). In the limit of infinite resolution, we would expect that $\tilde{T}$ be exactly zero at all radial locations.

In contrast to eigenmodes of the outer hyperbola in figure 11, the eigenmodes of the inner hyperbola arise from the class $\tilde{v}=\tilde{v}_{p}, \tilde{\rho}=\tilde{\rho}_{h}$. In this case, the homogeneous solution $\tilde{\rho}_{h}$ serves to drive a particular solution $\tilde{v}_{p}$, as is observed in the example in figure 22.

Finally, an example eigenmode of the portion of the continuous spectrum along the imaginary axis is displayed in figure 23. Like the eigenmodes of the outer hyperbolas, the imaginary axis modes derive from the solution class $\tilde{v}=\tilde{v}_{h}, \tilde{\rho}=0$. In the case of the imaginary axis branch cuts, however, $\tilde{v}_{h}=K_{1}\left(a_{2} r\right)$ (as opposed to $r^{2} K_{1}\left(a_{2} r\right)$ ) as $r \rightarrow \infty$. Perhaps because of the absence of the $r^{2}$ factor, these modes are allowed 

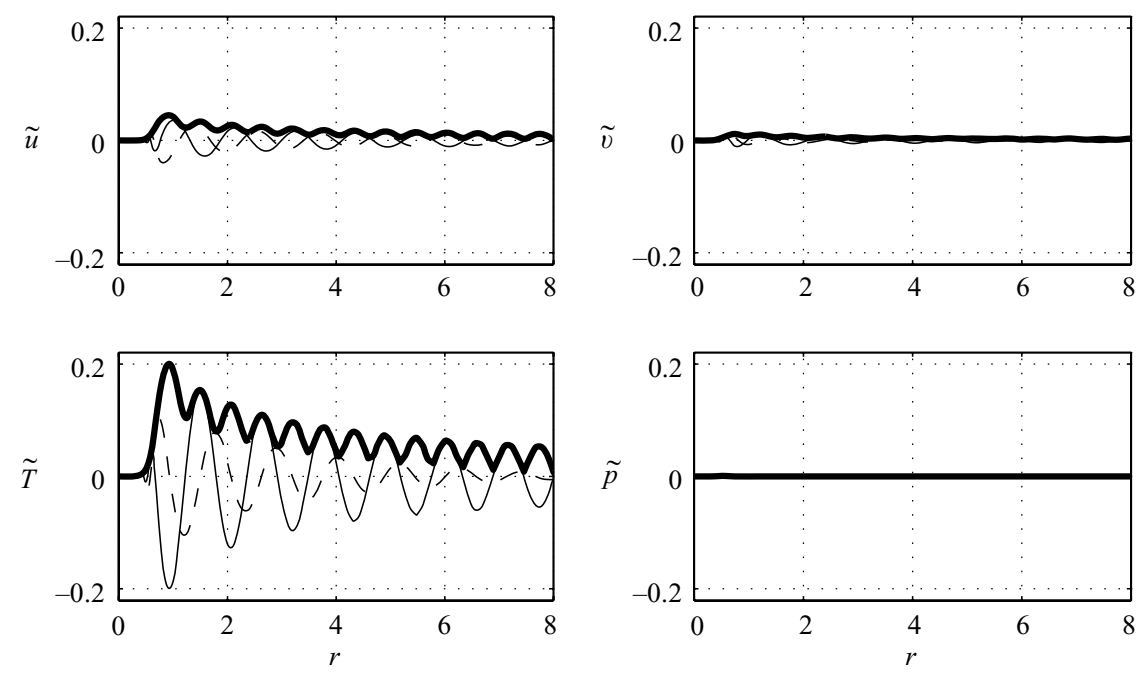

FIgURE 22. An example of an eigenmode of the continuous spectrum taken from the inner hyperbola branch cuts in figure 11. $\alpha=(13.952983,15.016015 \mathrm{i})$.
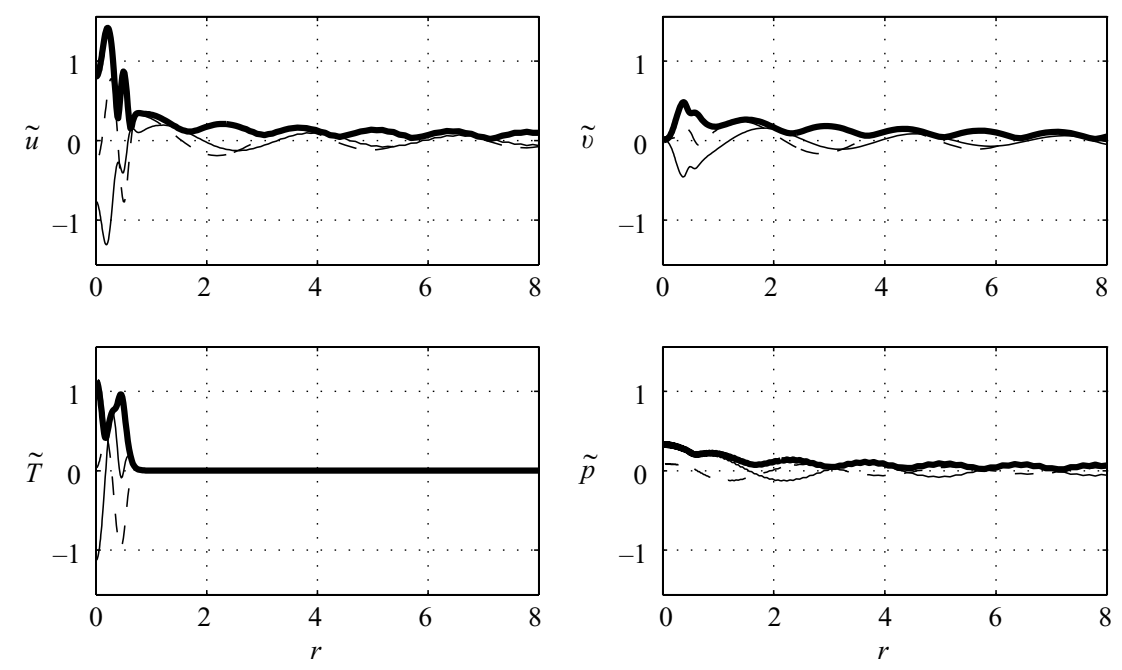

FIGURE 23. An example of an eigenmode of the continuous spectrum taken from the imaginary axis branch cuts in figure 11. $\alpha=(0.050173,2.301720 i)$.

to connect with oscillations in the interior of the jet. Since this is the case, we could hypothesize that the eigenmodes of the continuous spectrum along the imaginary axis play an important role in a free stream disturbance receptivity analysis. This might mean that the jet is particularly susceptible to disturbances of long axial wavelength, because for the imaginary axis modes, $\alpha_{r}$ is small.

In general, the continuous spectrum is necessary to ensure completeness of the linearized stability solution, which is essential when expansions into eigenfunctions become necessary or convenient. In addition, it is believed that the continuous spectrum plays an important role in receptivity studies when free stream disturbances interact with and excite internal (discrete) modes (see Jacobs \& Durbin 2001). 


\section{REFERENCES}

Ashris, D. E. \& Reshotкo, E. 1990 The vibrating ribbon problem revisited. J. Fluid Mech. 213, 531-547.

Bahadori, M. Y., Stocker, D. P., Vaughan, D. F., Zhou, L. \& Edelman, R. B. 1992 Effects of buoyancy on laminar, transitional, and turbulent gas jet diffusion flames. In Second International Workshop on Combustion Science in Microgravity. Cleveland, Ohio.

Batchelor, G. K. \& Gill, A. E. 1962 Analysis of the stability of axisymmetric jets. J. Fluid Mech. 14, 529-551.

Bird, R. W., Stewart, W. E. \& Lightfoot, E. N. 1960 Transport Phenomena. Wiley.

Boersma, B. J., Brethouwer, G. \& Nieuwstadt, F. T. M. 1998 A numerical investigation on the effect of the inflow conditions on the self-similar region of a round jet. Phys. of Fluids 10 (4), 899-909.

Brancher, P., Chomaz, J. M. \& Huerre, P. 1994 Direct numerical simulations of round jets: vortex induction and side jets. Phys. Fluids 6, 1768-1774.

BRIGGS, R. J. 1964 Electron-Stream Interaction with Plasmas. MIT Press.

Chomaz, J. M. 2005 Global instabilities in spatially developing flows: non-normality and nonlinearity. Annu. Rev. Fluid Mech. 37, 357-392.

Chorin, A. J. 1967 A numerical method for solving incompressible viscous flow problems. J. Comput. Phys. 2, 12-26.

Constantinescu, G. S. \& Lele, S. K. 2002 A highly accurate technique for the treatment of flow equations at the polar axis in cylindrical coordinates using series expansions. J. Comput. Phys. 183, 165-186.

Demare, D. \& Baillot, F. 2001 The role of secondary instabilities in the stabilization of a nonpremixed lifted jet flame. Phys. Fluids 13 (9), 2662-2670.

Drazin, P. G. \& ReID, W. H. 1981 Hydrodynamic Stability. Cambridge: University Press.

Durran, D. R. 1999 Numerical Methods for Wave Equations in Geophysical Fluid Dynamics. Springer.

Hegde, U., Zhou, L. \& BahadoRi, M. Y. 1994 The transition to turbulence of microgravity gas jet diffusion flames. Combust. Sci. Technol. 102, 95-113.

Huerre, P. 2000 Open shear flow instabilities. In Perspectives in Fluid Dynamics (ed. G. K. Batchelor, H. K. Moffatt \& M. G. Worster), pp. 159-229. Cambridge University Press.

Idicheria, C. A., Boxx, I. G. \& Clemens, N. T. 2003 Characteristics of turbulent nonpremixed jet flames in different gravity levels. In Third Joint Meeting of the US Sections of the Combustion Institute. Chicago, IL.

Jacobs, R. G. \& Durbin, P. A. 2001 Simulations of bypass transition. J. Fluid Mech. 428, 185-212.

Jendoubi, S. \& StRYKowski, P. J. 1994 Absolute and convective instability of axisymmetric jets with external flow. Phys. Fluids 6, 3000-3009.

Kaplan, C. R., Oran, E. S. \& Baek, S. W. 1994 Stabilization mechanism of lifted jet diffusion flames. In Twenty-Fifth Symp. (Int. on Combustion, pp. 1183-1189. The Combustion Institute, Pittsburgh.

Kyle, D. M. \& Sreenivasan, K. R. 1993 The instability and breakdown of a round variable-density jet. J. Fluid Mach. 249, 619-664.

Lele, S. K. 1992 Compact finite difference schemes with spectral-like resolution. J. Comput. Phys. $103,16-42$.

Lesshafft, L., Huerre, P., Sagaut, P. \& Terracol, M. 2006 Nonlinear global modes in hot jets. J. Fluid Mech. 554, 393-409.

MCMurTRY, P. A. 1987 Direct numerical simulations of a reacting mixing layer with chemical heat release. $\mathrm{PhD}$ thesis, University of Washington.

McMurtry, P. A., Jou, W. H., Riley, J. J. \& Metcalfe, R. W. 1986 Direct numerical simulations of mixing layers with heat release. AIAA J. 24, 962.

Michalke, A. 1984 Survey on jet instability theory. Prog. Aerospace Sci. 21, 159-199.

Mizobuchi, Y., Tachibana, S., Shinjo, J., Ogawa, S. \& Takeno, T. 2002 A numerical analysis of the structure of a turbulent hydrogen jet lifted flame. In Proc. Combustion Institute, pp. 2009-2015, Part 2. The Combustion Institute, Pittsburgh.

Monkewitz, P. A. \& Sohn, K. D. 1988 Absolute instability in hot jets. AIAA J. 26, 911-916.

Monkewitz, P. A., Bechert, D. W., Barsikow, B. \& Lehmann, B. 1990 Self-excited oscillations and mixing in a heated round jet. J. Fluid Mech. 213, 611-639. 
NaJM, H. N., Wyckoff, P. S. \& KNIO, O. M. 1998 A semi-implicit numerical scheme for reacting flow. I. Stiff chemistry. J. Comput. Phys. 143, 381-402.

Nichols, J. W. 2005 Simulation and stability analysis of jet diffusion flames. PhD thesis, University of Washington.

PierCe, C. D. \& Moin, P. 2001 Progress-variable approach for large-eddy simulation of turbulent combustion. Tech. Rep. Stanford University.

Poinsot, T. \& Veynante, D. 2001 Theoretical and Numerical Combustion. Edwards, Flourtown, PA.

Raynal, L., Harion, J.-L., Favre-Marinet, M. \& Binder, G. 1996 The oscillatory instability of plane variable-density jets. Phys. Fluids 8, 993-1006.

Schmid, P. J. \& Henningson, D. S. 2001 Stability and Transition in Shear Flows. Springer.

White, F. M. 1991 Viscous Fluid Flow, 2nd edn. McGraw-Hill. 